\title{
Reliable estimation of orbit errors in spaceborne SAR interferometry
}

\author{
The network approach
}

\author{
Hermann Bähr • Ramon F. Hanssen
}

Received: 9 May 2011 / Accepted: 21 April 2012 / Published online: 17 May 2012

(C) Springer-Verlag 2012

\begin{abstract}
An approach to improve orbital state vectors by orbit error estimates derived from residual phase patterns in synthetic aperture radar interferograms is presented. For individual interferograms, an error representation by two parameters is motivated: the baseline error in cross-range and the rate of change of the baseline error in range. For their estimation, two alternatives are proposed: a least squares approach that requires prior unwrapping and a less reliable gridsearch method handling the wrapped phase. In both cases, reliability is enhanced by mutual control of error estimates in an overdetermined network of linearly dependent interferometric combinations of images. Thus, systematic biases, e.g., due to unwrapping errors, can be detected and iteratively eliminated. Regularising the solution by a minimum-norm condition results in quasi-absolute orbit errors that refer to particular images. For the 31 images of a sample ENVISAT dataset, orbit corrections with a mutual consistency on the millimetre level have been inferred from 163 interferograms. The method itself qualifies by reliability and rigorous geometric modelling of the orbital error signal but does not consider interfering large scale deformation effects. However, a separation may be feasible in a combined processing with persistent scatterer approaches or by temporal filtering of the estimates.
\end{abstract}

Keywords InSAR - Baseline error · Orbit error . Network adjustment $\cdot$ Outlier test $\cdot$ Data snooping

H. Bähr $(\bowtie)$

Geodetic Institute, Karlsruhe Institute of Technology,

Englerstraße 7, 76131 Karlsruhe, Germany

e-mail: baehr@kit.edu

\section{R. F. Hanssen}

Geoscience and Remote Sensing, Delft University of Technology, Stevinweg 1, 2628 CN Delft, The Netherlands e-mail: R.F.Hanssen@ tudelft.nl

\section{Introduction}

Spaceborne repeat-pass synthetic aperture radar interferometry (InSAR) is a key technique for large-area deformation monitoring. Relative displacements of the ground occurring between two radar acquisitions are measured at pixel locations of two-dimensional SAR interferograms. These measurements can be biased by errors originating from a multitude of different sources. Each error source having its own characteristics, signals with different spatial or temporal properties are superposed to the signal of interest. When ground deformation is the signal of interest, it is the aim of InSAR processing to estimate and consecutively eliminate or mitigate all other signal contributions.

Inaccuracies in the satellite orbits affect interferograms in the form of an almost linear signal and scale the height ambiguity. Whereas this latter effect is usually negligible and orbit errors may be disregarded for localised phenomena, their effect can be considerable if the deformation regime covers a larger area. A common approach to separate the signal components from each other is to identify the orbital contribution by its long spatial wavelength. Whereas this discrimination is efficient for a large number of applications, it does not apply in the presence of a large-scale deformation signal like tectonic movement or tides that has similar spatial characteristics. In this case, part of the deformation may be misinterpreted as orbit error.

The most popular method to account for orbit errors is the subtraction of an estimated linear trend (or phase ramp) from the interferogram. This is easy to implement but involves a minor bias, since the orbital error signal is not rigorously linear. This flaw is overcome by more sophisticated methods that do not correct the interferometric phase for the effect of orbit errors but rather modify the satellite trajectories so that the error signal dissolves. Massonnet and Feigl (1998) 
as well as Kohlhase et al. (2003) deduce the required modifications from the fringe count along the edges of an interferogram. Following their approaches, orbit corrections can be estimated from phase differences or gradients that have been measured between defined pixel locations.

When deriving orbit errors from residual fringe patterns in the interferometric phase, only relative orbit corrections can be inferred, adjusting the trajectories of the two acquisitions with reference to each other. Hence, orbit error estimation from one interferogram alone is equivalent to the estimation of baseline errors, where the interferometric baseline is defined as the difference vector of the sensor positions of master and slave acquisition. A lot of research has been pursued in this domain with different parameterisations of the three-dimensional, time-dependent baseline vector in its across-track and radial component (Small et al. 1993; Werner et al. 1993; Zhang et al. 2009). Even though these approaches perform well in mitigating the effect of orbital errors, little attention has been paid on an optimal parameterisation of the baseline to avoid physically unlikely estimates.

The present contribution starts with a brief review on quality of orbit products and a sensitivity analysis of the baseline with respect to the interferometric phase. Based thereupon, a representation of baseline errors by two parameters is motivated, namely the baseline component in cross-range and the rate of change of the component in range direction. In Sect. 3, a least squares approach (Bähr and Hanssen 2010) is proposed to estimate the baseline error from the unwrapped interferometric phase. To overcome the requirement of unwrappable interferograms, also an alternative method is presented that can handle the wrapped phase but is considered less reliable. It is similar to the periodogram approach, where the dominant fringe frequency is determined by Fourier analysis to deduce baseline components (Singh et al. 1997; Monti Guarnieri et al. 2000; Bing et al. 2006). By contrast, it does not imply linearity of the orbital error signal and accounts for variations in the topographic height, involving rigorous geometric modelling.

A crucial issue in baseline error estimation is to ensure reliability, since interferograms with suboptimal coherence may be contaminated by unwrapping errors. A promising approach to cross-check the error estimate of the baseline between two acquisitions is to exploit linear combinations of interferograms with different perpendicular and temporal baselines. This concept can be regarded as setting up a network of interferograms that connect the available images on redundant paths in this spatio-temporal baseline-space. Thus, quasi-absolute orbit errors can be inferred that either refer to a global master or are, more conveniently, defined by a minimum-norm condition (Kohlhase et al. 2003; Biggs et al. 2007; Bähr and Hanssen 2010). Furthermore, inconsistencies that are due to interferogram-specific filtering are adjusted, enhancing the precision of the baseline error estimates.
Offering a mechanism to detect outliers due to unwrapping, the network approach provides a notable gain in reliability, whereas an increased processing load is required. In Sect. 4, the method from Bähr and Hanssen (2010) is evaluated for its capability to detect unwrapping errors. Further considerations address the optimality of stochastic modelling.

Even with the correction approaches listed above, the estimation of large-scale deformation signals is cumbersome, as both orbit errors and gradient atmospheric propagation delays can induce signals with similar spatial characteristics. However, it is not the aim of this contribution to tackle the separability of the orbital error signal from other signal components. It is rather intended to provide a reliable method to eliminate the orbital contribution with as little userinteraction as possible. Nevertheless, some thoughts are also offered in Sect. 5 on the mutual bias of orbit correction, deformation and the atmospheric signal contribution and its mitigation.

\section{Orbit errors}

In order to motivate an effective correction methodology, this section is dedicated to a both quantitative and qualitative analysis of satellite orbit errors and their effects on interferograms. After reviewing available quality information on orbit products of past and current SAR missions, the mutual sensitivity of the interferometric phase and orbit errors is analysed. Finally, an appropriate parameterisation is proposed.

\subsection{Accuracy of orbit products}

Precise orbits of SAR satellites are determined by space geodetic techniques. Earlier missions like the European Remote Sensing Satellites (ERS-1/2) made use of Satellite Laser Ranging (SLR), supported by radar altimetry measurements. At Delft University of Technology, a root mean square (RMS) error of $4 \mathrm{~cm}$ for the radial component of ERS orbit solutions could be attained (Doornbos and Scharroo 2005). The accuracy of the along- and across-track components is definitely worse, which is suggested by differences of the order of $15 \mathrm{~cm}$ for solutions based on different gravity models (Scharroo and Visser 1998). For the likewise SLR-tracked Environmental Satellite (ENVISAT), the DORIS system (Doppler Orbitography and Radiopositioning Integrated by Satellite) provides complementary measurements. The accuracy of the best orbit products is estimated to be $3 \mathrm{~cm}$ in the radial component and $10 \mathrm{~cm}$ in 3D (Otten and Dow 2005). More recent missions rely primarily on the Global Positioning System (GPS) for orbit determination, supported by SLR. For TerraSAR-X, the RMS orbit accuracy has been assessed to be at the $2 \mathrm{~cm}$ level (Yoon et al. 2009). Comparing the GPS orbits of the Advanced Land Observing Satellite (ALOS) to SLR 
solutions yielded RMS deviations of overlapping arcs between 2 and $15 \mathrm{~cm}$ (Nakamura et al. 2007), assumedly in 3D.

It must be stressed that these quality indicators have to be interpreted with care, since a rigorously independent validation is not possible. Subsequent arcs or solutions from different analysis centres rely at least partially on the same data. In addition, systematic errors in atmospheric and force models may affect all measurements in the same way, even when different techniques are involved. Furthermore, global quality measures are not necessarily stationary in time and space. There may be local quality variations due to an inhomogeneous distribution of SLR tracking stations. An increased solar activity or bad atmospheric conditions can entail less accurate solutions. In some cases, accuracy assessments only consider selected epochs, which are not necessarily representative for the whole mission.

More conservative quality estimates are less optimistic. Yoon et al. (2009) assess the precise scientific TerraSAR-X orbits to be "definitely better than $10 \mathrm{~cm}$ ". For some arc overlaps of ALOS orbits, deviations exceed $30 \mathrm{~cm}$ (Nakamura et al. 2007). Official requirement specifications for ALOS range up to $1 \mathrm{~m}$ (European Space Agency 2007). Radarsat-2 orbits have a nominal $3 \sigma$ accuracy of even $15 \mathrm{~m} .{ }^{1}$

This illustrates that even though accuracies of orbit products from recent missions are estimated to be on the centimetre level, possible deviations from the true trajectory of several decimetres may occur. For Radarsat, the expectable accuracy is even worse.

\subsection{Parameterisation}

Orbit products describe the satellite's trajectory by state vectors, which subsume positions and velocities at distinct epochs. The interferometric baseline is represented by the difference vector of the sensor positions $\vec{x}$ from two acquisitions, referred to as master $(\mathrm{M})$ and slave $(\mathrm{S})$ :

$\vec{B}(t)=\vec{x}_{\mathrm{S}}(t)-\vec{x}_{\mathrm{M}}(t)$.

It is decomposable into its horizontal (across-track), alongtrack and vertical (radial) component $B_{h}(t), B_{a}(t)$ and $B_{v}(t)$, respectively. Considering only the two-dimensional $\left(B_{h}, B_{v}\right)$-plane, there are alternative ways of baseline decomposition (see Fig. 1). The baseline can equivalently be described by its length $B=|\vec{B}|$ and its orientation angle $\alpha$. A third representation consists of its parallel component $B_{\|}$in range and perpendicular component $B_{\perp}$ in cross-range direction, respectively (Hanssen 2001):

\footnotetext{
${ }^{1}$ MDA Corporation, http://www.radarsat2.info/about/mission.asp, accessed on $10 \mathrm{Feb} 2011$.
}

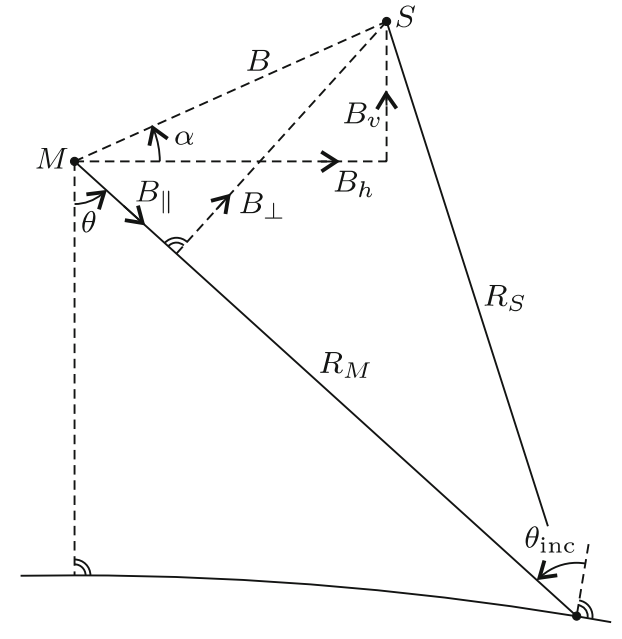

Fig. 1 Decomposition of the interferometric baseline in a plane perpendicular to the flight direction. The representations by its horizontal and vertical component ( $B_{h}$ and $B_{v}$ ) or its length and orientation angle ( $B$ and $\alpha$ ) are unambiguous, whereas the description by parallel (range) and perpendicular (cross-range) component $\left(B_{\|}\right.$and $B_{\perp}$ ) depends on range. So do the look angle $\theta$ and the local incidence angle $\theta_{\text {inc }}$

$$
\begin{aligned}
B_{\|}(t, \theta) & =B_{h}(t) \sin \theta-B_{v}(t) \cos \theta \\
& =B(t) \sin (\theta-\alpha(t)) \\
B_{\perp}(t, \theta) & =B_{h}(t) \cos \theta+B_{v}(t) \sin \theta \\
& =B(t) \cos (\theta-\alpha(t)) .
\end{aligned}
$$

The baseline can be related to the interferometric phase by the following approximation:

$\phi(t, R)=-\frac{4 \pi}{\lambda}\left(R_{M}(t)-R_{S}(t)\right) \approx-\frac{4 \pi}{\lambda} B_{\|}(t, R)$,

where $\lambda$ is the radar wavelength. $R_{M}=: R$ and $R_{S}$ are the respective range measurements of master and slave. In order to assess the sensitivity of an interferogram to baseline errors, the residual phase $d \phi \approx-\frac{4 \pi}{\lambda} d B_{\|}$is now considered as a function of an error baseline $d \vec{B}$ that is superposed to the interferometric baseline. Taylor series expansion in azimuth time $t$ and look angle $\theta(R)$ yields with Eq. (2):

$$
\begin{aligned}
& d \phi(t, \theta)=-\frac{4 \pi}{\lambda}\left(d B_{\|}\left(t_{0}, \theta_{0}\right)+d \dot{B}_{\|}\left(t_{0}, \theta_{0}\right) d t\right. \\
& \quad+d B_{\perp}\left(t_{0}, \theta_{0}\right) d \theta+\frac{1}{2} d \ddot{B}_{\|}\left(t_{0}, \theta_{0}\right) d t^{2} \\
& \left.+d \dot{B}_{\perp}\left(t_{0}, \theta_{0}\right) d t d \theta-\frac{1}{2} d B_{\|}\left(t_{0}, \theta_{0}\right) d \theta^{2}+\cdots\right) .
\end{aligned}
$$

The zero-order term can be ignored; a constant phase change to the whole interferogram does not affect its interpretation, since InSAR is a relative technique. The first-order terms are characterised by the baseline error components $d \dot{B}_{\|}$and $d B_{\perp}$, which result in linear fringes in azimuth and range, respectively (see Fig. 2a-d). By rule of thumb, a rough estimate of the baseline error can be obtained by multiplying the number of fringes in azimuth or range by the respective conversion factors: 


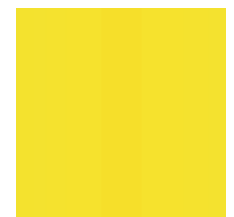

(a) $d B_{\|}=26 \mathrm{~cm}$

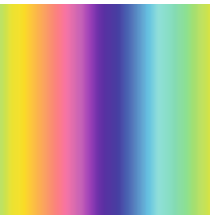

(b) $d B_{\perp}=26 \mathrm{~cm}$

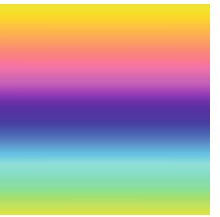

(c) $d \dot{B}_{\|}=1.7 \frac{\mathrm{mm}}{\mathrm{s}}$

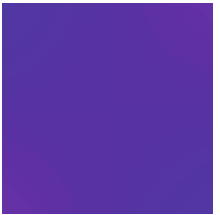

(d) $d \dot{B}_{\perp}=1.7 \frac{\mathrm{mm}}{\mathrm{s}}$

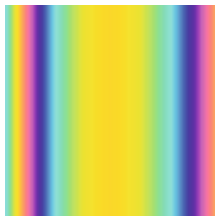

(e) $d B_{\|}=21 \mathrm{~m}$

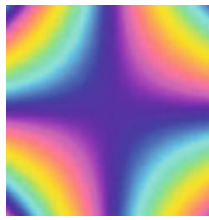

(f) $d \dot{B}_{\perp}=6.7 \frac{\mathrm{cm}}{\mathrm{s}}$
Fig. 2 Error signals induced into the interferometric phase by errors in different baseline components, assuming $\lambda=5.7 \mathrm{~cm}$ and a mean look angle of $20^{\circ}$ (ERS). The decomposition into $B_{\|}$and $B_{\perp}$ is defined by the line of sight to the centre of the scene of $100 \times 100 \mathrm{~km}^{2}$ size. (a, b) The effect of an error in $B_{\|}$is negligible compared to an error of the same amount in $B_{\perp}$. (c, d) An analogous conclusion can be drawn for $\dot{B}_{\perp}$ and $\dot{B}_{\|}$. (e, f) Errors in $B_{\|}$and $\dot{B}_{\perp}$ must be disproportionately large to yield a distinct interferometric signal (Bähr and Hanssen 2010)

$d \dot{B}_{\|, 2 \pi}=-\frac{\lambda}{2 \Delta t}, \quad d B_{\perp, 2 \pi}=-\frac{\lambda}{2 \Delta \theta}$,

where $\Delta t$ is the total acquisition time and $\Delta \theta$ is the range of look angles, respectively, for the whole scene. Individual computations of these numbers for some missions are listed in Table 1.

With the objective of mitigating the effect of baseline errors as far as possible, a suitable parameterisation is required. In the following, a number of options will be evaluated, considering the error signals $d \phi(t)$ in azimuth and $d \phi(R)$ in range separately.

Starting with the signal in range, Fig. 3a shows how the maximum bias of the interferometric measurement due to an error baseline $d \vec{B}$ of constant length $d B$ depends on its orientation. The extrema at a mean look angle $\theta_{0}$ plus multiples of $90^{\circ}$ suggest a further consideration of the representation by $d B_{\|}\left(\theta_{0}\right)$ and $d B_{\perp}\left(\theta_{0}\right)$. Estimating both parameters from the residual phase of an interferogram may yield geometrically unrealistic estimates. As errors in $B_{\|}$induce only a small signal in the phase (see Fig. 2a), small large-scale variations in the atmospheric propagation delay can conversely cause estimates for $d B_{\|}$on the metre level. In a reverse conclusion, it would be a tolerable loss to constrain $d B_{\|}$to zero, given the little effect this parameter has on the phase.

Figure $3 \mathrm{~b}$ displays the approximation error that is made by ignoring errors in $B_{\|}$. It further compares the performance of a $d B_{\perp}$-only parameterisation to alternative parametric models, the most common of which is a polynomial in range:

$p_{n}(R)=\sum_{k=1}^{n} a_{k} R^{k}$

It can be seen from the figure that the $d B_{\perp}$-parameterisation performs three times better than the common approach of a "linear phase ramp" $p_{1}(R)$. A third approximation model to be pointed out in this context is a polynomial in look angle:

$p_{n}(\theta)=\sum_{k=1}^{n} a_{k} \theta^{k}$

As $\partial \theta / \partial R$ is not constant, the performance of the approximation by $p_{n}(\theta)$ is different from that by $p_{n}(R)$. Application requires an individual computation of $\theta(R)$ for every range pixel, but this is still easier to implement than the parameterisation by baseline components. The linear model $p_{1}(\theta)$ performs even slightly better than $d B_{\perp}$, whereas the difference between the two is considered negligible. Two-parametric models like $p_{2}(R), p_{2}(\theta)$ or $\left(d B_{\|}, d B_{\perp}\right)$ would reduce the approximation error even more but have the disadvantage that they may distort a possible large-scale deformation signal more seriously. It has to be carefully investigated from case to case if the increased accuracy of two-parametric models outweighs this deficiency.

Table 1 Baseline errors $d \dot{B}_{\|, 2 \pi}$ and $d B_{\perp, 2 \pi}$ inducing exactly one fringe in azimuth or range, respectively, evaluated for different sensors. $H_{\text {sat }}$ is the mean height of the platform

\begin{tabular}{|c|c|c|c|c|c|c|c|c|}
\hline Sensor & Mode & $\lambda(\mathrm{cm})$ & $H_{\text {sat }}(\mathrm{km})$ & $\theta\left({ }^{\circ}\right)$ & $\theta_{\text {inc }}\left({ }^{\circ}\right)$ & Scene $(\mathrm{km}) \times(\mathrm{km})$ & $d \dot{B}_{\|, 2 \pi}(\mathrm{mm} / \mathrm{s})$ & $d B_{\perp, 2 \pi}(\mathrm{cm})$ \\
\hline ALOS PALSAR & FBS 7 & 23.6 & 692 & $32-36$ & $36-41$ & $70 \times 70$ & 11.4 & 185 \\
\hline ENVISAT ASAR & IS2 & 5.6 & 800 & $17-23$ & $19-26$ & $100 \times 100$ & 1.9 & 26 \\
\hline ERS-1/2 & & 5.7 & 790 & $17-23$ & $20-27$ & $100 \times 100$ & 1.9 & 26 \\
\hline Radarsat-1/2 & S3 & 5.6 & 798 & $26-32$ & $30-36$ & $100 \times 100$ & 1.8 & 31 \\
\hline Sentinel-1 & IWS & 5.6 & 693 & $22-37$ & $25-42$ & $170 \times 250$ & 1.1 & 11 \\
\hline TerraSAR-X & strip_003 & 3.1 & 514 & $18-21$ & $20-23$ & $50 \times 32$ & 2.2 & 29 \\
\hline TerraSAR-X & strip_010 & 3.1 & 514 & $33-35$ & $36-39$ & $50 \times 32$ & 2.2 & 39 \\
\hline TerraSAR-X & strip_014 & 3.1 & 514 & 39-41 & $43-46$ & $50 \times 32$ & 2.2 & 48 \\
\hline
\end{tabular}

Sensor parameters follow the nominal specifications where available. Note that these numbers do not apply precisely on any individual data product. $d \dot{B}_{\|, 2 \pi}$ and $d B_{\perp, 2 \pi}$ may vary slightly as the case arises 
(a) Orbital Error Signal

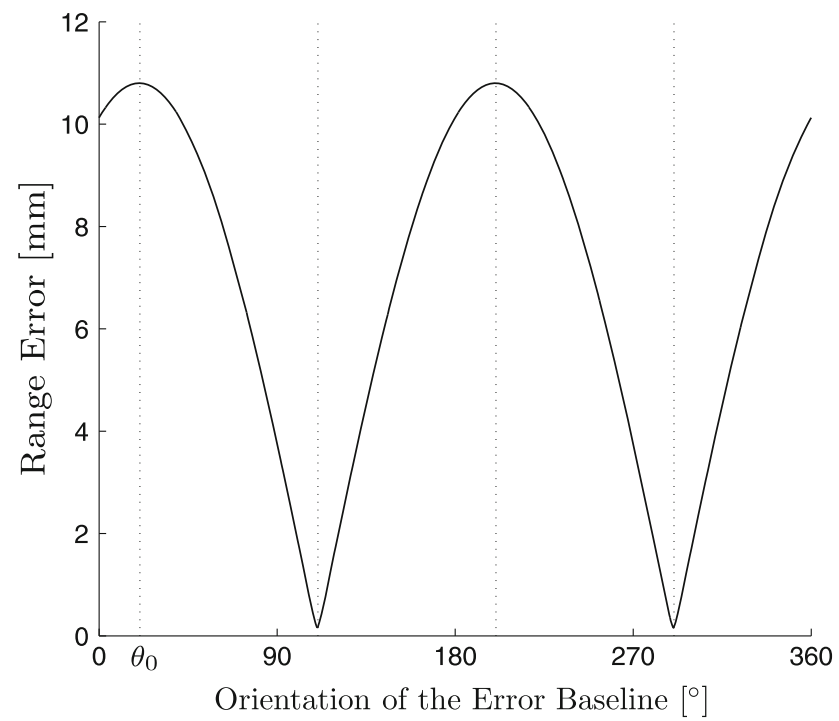

Fig. 3 Characteristics of a simulated orbital error signal and performance of an approximation thereof. The simulations are based on an error baseline of $d B=1 \mathrm{dm}$ length with varying orientation with respect to the horizontal. Sensor height and field of view for a full ERS scene have been assumed. The plotted range errors are defined as maximum minus minimum bias of the interferometric measurement and (b) Approximation Error

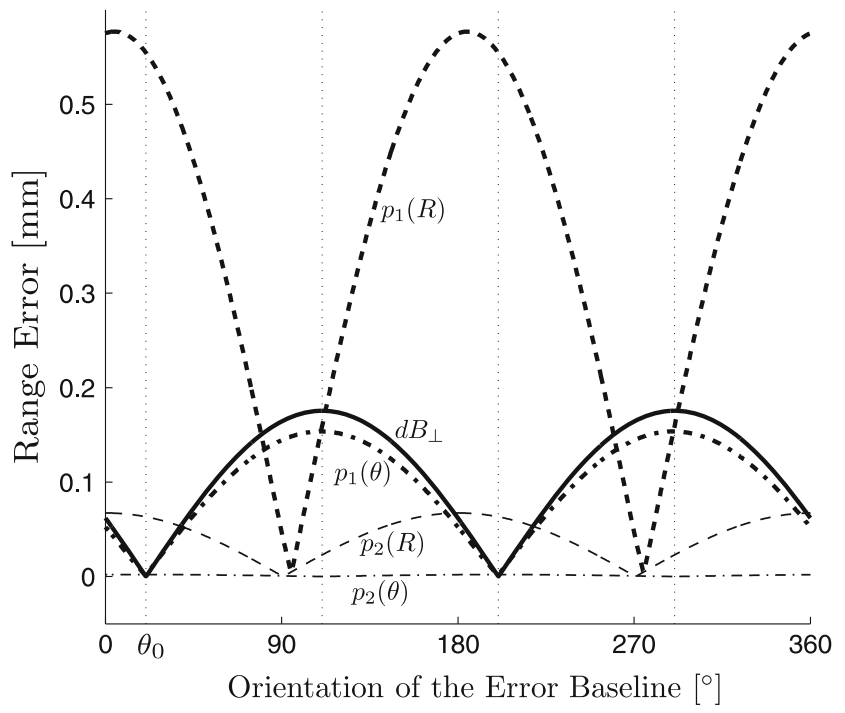

scale almost linearly with $d B$. Qualitatively, the results are conferrable to other sensors. For a quantitative evaluation, please refer to Table 2 . a Range error of the orbital error signal itself with respect to zero phase. b Approximation error of the orbital error signal for different parametric models as explained in the text

Table 2 Maximum residual range error due to approximation of an orbital error signal in range direction, induced by an error baseline of $1 \mathrm{dm}$ length for different sensors and parametric models

\begin{tabular}{|c|c|c|c|c|c|c|c|c|}
\hline \multirow[t]{2}{*}{ Sensor } & \multirow[t]{2}{*}{ Mode } & \multicolumn{7}{|c|}{ Maximum residual range error (mm) } \\
\hline & & $p_{0}$ & $p_{1}(R)$ & $p_{1}(\theta)$ & $\left(d B_{\perp}\right)$ & $p_{2}(R)$ & $p_{2}(\theta)$ & $\left(d B_{\|}, d B_{\perp}\right)$ \\
\hline ALOS PALSAR & FBS 7 & 6.4 & 0.17 & 0.05 & 0.06 & 0.01 & 0.00 & 0 \\
\hline ENVISAT ASAR & IS2 & 10.7 & 0.58 & 0.15 & 0.17 & 0.07 & 0.00 & 0 \\
\hline ERS-1/2 & & 10.8 & 0.58 & 0.15 & 0.18 & 0.07 & 0.00 & 0 \\
\hline Radarsat- $1 / 2$ & $\mathrm{~S} 3$ & 9.0 & 0.35 & 0.11 & 0.12 & 0.03 & 0.00 & 0 \\
\hline Sentinel-1 & IWS & 25.3 & 2.91 & 0.89 & 1.15 & 0.58 & 0.03 & 0 \\
\hline TerraSAR-X & strip_003 & 5.4 & 0.14 & 0.04 & 0.04 & 0.01 & 0.00 & 0 \\
\hline TerraSAR-X & strip_010 & 4.0 & 0.07 & 0.02 & 0.02 & 0.00 & 0.00 & 0 \\
\hline TerraSAR-X & strip_014 & 3.3 & 0.05 & 0.01 & 0.01 & 0.00 & 0.00 & 0 \\
\hline
\end{tabular}

See also Fig. 3b. $p_{0}$ stands for the uncorrected error signal itself, see Fig. 3a

To support the choice of an adequate model, Table 2 lists the maximum approximation error for different sensors and modes, assuming an error baseline of constant length $d B=$ $1 \mathrm{dm}$. All numbers can be adapted to bigger or smaller baseline errors, since the phase error scales linearly with $d B$. This can be seen from Eq. (4) under consideration of Eq. (2).

For the orbital error signal in range can be concluded that in case of usual orbit errors below $1 \mathrm{dm}$ a $p_{1}(R)$-correction is sufficient. For higher errors, one of the other one-parametric models should be considered. This applies especially to Radarsat- 2 and even more to Radarsat- 1 , where the orbit data are generally more inaccurate. Moreover, for the planned mission Sentinel-1, where the swath width in the Interferometric Wide Swath Mode is designed to be $250 \mathrm{~km}$, it is advisable to rely on either $p_{1}(\theta)$ or the $d B_{\perp}$-parameterisation. In the present article, the $d B_{\perp}$-approach is preferred for its more generic nature. For the reasons just given, models involving more than one parameter to describe the dependence of phase on range are not considered practical.

The error signal in azimuth is a direct translation of variations in $d B_{\|}$into the interferometric phase. A constant rate of change $d \dot{B}_{\|}$induces a perfectly linear error signal 
(see Fig. 2c). Generally, any phase pattern $d \phi(t)$ could result from arbitrarily varying baseline errors $d B_{\|}(t)$. However, as orbital trajectories have the nature to be very smooth curves, it is unlikely that errors in these curves undergo completely random variations. Therefore, a linearly varying error in the parallel baseline component is considered an appropriate parameterisation of the baseline error for the short acquisition time of a single radar scene. The benefit of higher order polynomials is questionable, because nonlinear components would rather be dominated by atmospheric effects, yielding unrealistically high estimates. Nevertheless, if long data takes are processed as a single frame, a quadratic polynomial in $d B_{\|}(t)$ may be considerable.

It can be concluded that for a wide majority of applications, baseline errors are appropriately parameterised by $d \dot{B}_{\|}$ and $d B_{\perp}$. This representation will be adopted for the following considerations.

\section{Methodology}

To estimate orbit errors for $m$ acquisitions, a network of $n \geq m$ interferograms is set up in the domains of $B_{\perp}$ and time (for an example see Fig. 6). Starting point is the residual interferometric phase, where "residual" refers to the assumption that the orbital effect is the only remaining signal after removal of reference and topographic phase from the interferograms. In a first step, two baseline error parameters $\mathbf{b}_{\theta}^{T}=\left(d \dot{B}_{\|}, d B_{\perp}\right)$ per interferogram are inferred from the residual phases of selected pixels $\phi^{T}=\left(\ldots, d \phi_{i}, \ldots\right)$, where unwrapping is only required for the first of two approaches that are described in the following. Secondly, network misclosures in the $2 n$ baseline error parameters $\mathbf{y}^{T}=\left(\ldots, d \dot{B}_{\|, k}, d B_{\perp, k}, \ldots\right)$ of all interferograms are adjusted. The resulting $2 m$ acquisition-related orbit errors $\mathbf{x}^{T}=\left(\ldots, d \dot{x}_{\|, i}, d x_{\perp, i}, \ldots\right)$ are defined by a minimumnorm condition and can be used to correct the orbit trajectories at hand for re-processing.

\subsection{Baseline error estimation from the unwrapped phase}

The estimation of orbit errors from the unwrapped residual interferometric phase $d \phi$ by the method of least squares is based on the observation equation:

$\mathrm{E}\{d \phi\}=-\frac{4 \pi}{\lambda}\left(R_{M}-R_{S}\right)+\phi_{0}$.

$\phi_{0}$ is a constant phase shift that has to be introduced to account for the inferior precision of range measurement compared with phase measurement. Linearising this relation with respect to the horizontal, along-track and vertical components of the orbit positions of master and slave yields:

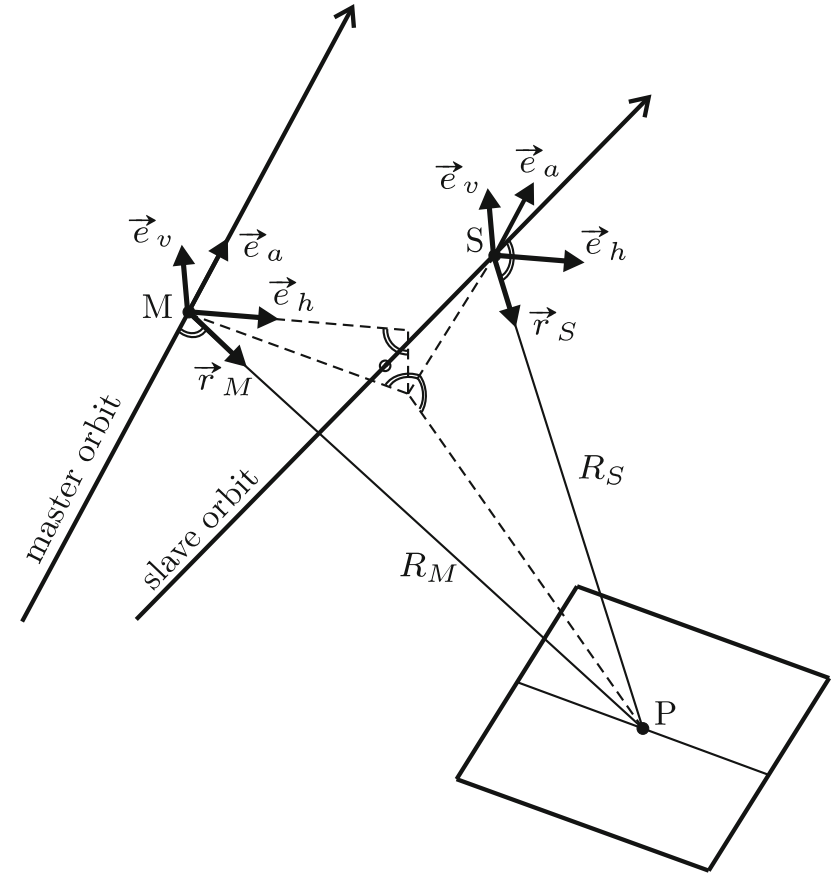

Fig. 4 Virtual acquisition geometry for non-parallel orbits that can be assumed in case of zero-Doppler focussed data

$$
\begin{aligned}
\mathrm{E}\{d \phi\}= & -\frac{4 \pi}{\lambda}\left(\vec{r}_{M} \cdot \vec{e}_{h} d x_{h, M}+\vec{r}_{M} \cdot \vec{e}_{a} d x_{a, M}\right. \\
& +\vec{r}_{M} \cdot \vec{e}_{v} d x_{v, M}-\vec{r}_{S} \cdot \vec{e}_{h} d x_{h, S} \\
& \left.-\vec{r}_{S} \cdot \vec{e}_{a} d x_{a, S}-\vec{r}_{S} \cdot \vec{e}_{v} d x_{v, S}\right)+\phi_{0},
\end{aligned}
$$

where $\vec{r}_{M}(t, \theta)$ and $\vec{r}_{S}(t, \theta)$ are unit vectors describing the line of sight. $\vec{e}_{h}(t), \vec{e}_{a}(t)$ and $\vec{e}_{v}(t)$ are unit vectors in horizontal, along-track and vertical direction, forming a Frenet frame (see Fig. 4). As long as only one interferogram on its own is considered, this frame can be conventionally defined by the master orbit. Note that all coefficients in Eq. (9) are also calculable in case of variable but known topography. For small squint angles, as they occur in spaceborne SAR, it follows from $\vec{r}_{M} \cdot \vec{e}_{a} \approx 0$ and $\vec{r}_{S} \cdot \vec{e}_{a} \approx 0$ that the interferometric phase is not sensitive to orbit errors in along-track direction. Consequently, these components are not considered any further.

Moreover, the coefficients in Eq. (9) that correspond to $d x_{h}$ and $d x_{v}$ are almost identical for master and slave due to the very small divergence between $\vec{r}_{M}$ and $\vec{r}_{S}$. This makes the joint estimation of individual orbit errors for both master and slave an ill-posed problem. Considering one interferogram on its own, only a baseline error $d \vec{B}=d \vec{x}_{S}-d \vec{x}_{M}$ can be robustly estimated. In this case, it must be decided if the estimated error is attributed to inaccuracies in the master orbit, the slave orbit or to errors in both of them. In the following, the error is attributed in equal proportions to master and slave in order to avoid an arbitrary discrimination of one of the two acquisitions. Additionally allowing for a linear 
temporal variation of the baseline components, the altered model reads:

$$
\begin{aligned}
\mathrm{E}\{d \phi\}= & \frac{4 \pi}{\lambda}\left[\frac{\vec{r}_{M}+\vec{r}_{S}}{2} \cdot \vec{e}_{h}\left(d B_{h}+t d \dot{B}_{h}\right)\right. \\
& \left.+\frac{\vec{r}_{M}+\vec{r}_{S}}{2} \cdot \vec{e}_{v}\left(d B_{v}+t d \dot{B}_{v}\right)\right]+\phi_{0} .
\end{aligned}
$$

Considering the residual interferometric phases $\boldsymbol{\phi}^{T}=$ $\left(\ldots, d \phi_{i}, \ldots\right)$ of $n_{\phi}$ pixels that are regularly arranged on a grid spanning the whole interferogram, baseline parameters $\mathbf{b}^{T}=\left(d B_{h}, d \dot{B}_{h}, d B_{v}, d \dot{B}_{v}\right)$ can be estimated in a functional model of the following kind:

$$
\mathrm{E}\{\boldsymbol{\phi}\}=\mathbf{A}_{b} \mathbf{b}+\mathbf{1} \phi_{0},
$$

where $\mathbf{A}_{b}$ is the design matrix and $\mathbf{1}^{T}=(1,1, \ldots, 1)$. The stochastic model is generically defined by some covariance matrix:

$$
\mathrm{D}\{\boldsymbol{\phi}\}=\sigma_{0}^{2} \mathbf{Q}_{\phi},
$$

the choice of which will be discussed in detail in Sect. 4.1.

Similar approaches have been proposed by other authors. Small et al. (1993) used relation (10) to estimate $d B_{h}, d \dot{B}_{h}$ and $\phi_{0}$, attributing baseline errors to the master orbit only. In Werner et al. (1993), the parameter set was extended by $d B_{v}$ as a fourth unknown. Kohlhase et al. (2003) avoided the arbitrary choice to decide in what proportions baseline errors are attributed to master and slave by evaluating relation (9) in the network context (see Sect. 4.4). Using phase differences as basic observations instead of unwrapped phase values, they estimated $d x_{h, i}\left(t_{j}\right)$ and $d x_{v, i}\left(t_{j}\right)$ per image $i$ for two distinct azimuth times $t_{j}$ and derived rates of change by differencing. Whereas the shift parameter $\phi_{0}$ cancels out in their approach, it is not straightforward to consider phase differences deduced from common phase observations in the stochastic model. Therefore, the present contribution rather relies on the original unwrapped phases as observations.

As $\phi_{0}$ is of no further interest, it can be eliminated from Eq. (11), yielding (Teunissen 2000):

$$
\mathrm{E}\{\boldsymbol{\phi}\}=\overline{\mathbf{A}}_{b} \mathbf{b}
$$

with:

$$
\overline{\mathbf{A}}_{b}=\left(\mathbf{I}-\mathbf{1}\left(\mathbf{1}^{T} \mathbf{Q}_{\phi}^{-1} \mathbf{1}\right)^{-1} \mathbf{1}^{T} \mathbf{Q}_{\phi}^{-1}\right) \mathbf{A}_{b} .
$$

The relative estimation quality of the parameters $\hat{\mathbf{b}}$ is given by their covariance matrix:

$$
\mathrm{D}\{\hat{\mathbf{b}}\}=\sigma_{0}^{2}\left(\overline{\mathbf{A}}_{b}^{T} \mathbf{Q}_{\phi}^{-1} \overline{\mathbf{A}}_{b}\right)^{-1}
$$

and can be visualised qualitatively by the error ellipses in Fig. 5. These are strongly elongated, showing that the baseline is determined best perpendicular to the look direction, whereas its rate of change has maximum precision in look

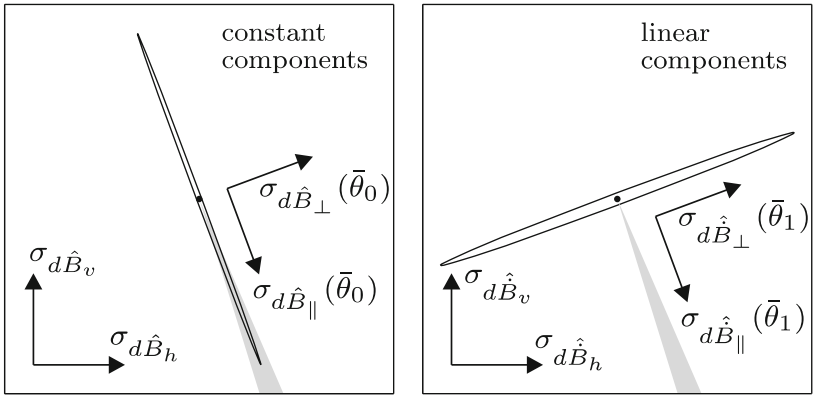

Fig. 5 Relative estimation quality of constant and linear components of the baseline error, visualised by error ellipses. The grey area represents the sensor's field of view. The orientation angles of the ellipses $\bar{\theta}_{0}$ and $\bar{\theta}_{1}$, respectively, can be computed from the eigenspaces of the corresponding covariance matrix. It follows that the estimability of $d B_{\perp}\left(\bar{\theta}_{0}\right)$ and $d \dot{B}_{\|}\left(\bar{\theta}_{1}\right)$ is good, whereas $d B_{\|}\left(\bar{\theta}_{0}\right)$ and $d \dot{B}_{\perp}\left(\bar{\theta}_{1}\right)$ are only weakly determined

direction. This basically confirms the previously drawn conclusions identifying $d \dot{B}_{\|}$and $d B_{\perp}$ as the components with the most significant effect on the interferometric phase.

Even though the complementary components $d B_{\|}$and $d \dot{B}_{\perp}$ are theoretically estimable, the estimates would be too weakly determined to be considered reliable. This can be seen from Fig. 2e, f, where a relatively huge error in $B_{\|}$induces only a very faint error signal in the phase. Conversely, a faint atmospheric signal that matches by chance this phase pattern, would result in unrealistically high estimates of $d B_{\|}$in the order of metres. Analogous considerations apply to $d \dot{B}_{\perp}$. Therefore, it is preferable to constrain these two components to zero. This is achieved by confining the parameter space from four parameters $\mathbf{b}$ to two parameters $\mathbf{b}_{\theta}^{T}=\left(d \dot{B}_{\|}, d B_{\perp}\right)$ :

$\mathrm{E}\{\boldsymbol{\phi}\}=\overline{\mathbf{A}}_{b} \mathbf{T}^{T} \mathbf{b}_{\theta}$

with:

$\mathbf{T}=\left(\begin{array}{cccc}0 & \sin \left(\theta_{0}\right) & 0 & -\cos \left(\theta_{0}\right) \\ \cos \left(\theta_{0}\right) & 0 & \sin \left(\theta_{0}\right) & 0\end{array}\right)$.

The mean look angle $\theta_{0}=\left(\bar{\theta}_{0}+\bar{\theta}_{1}\right) / 2$, which is required for the decomposition into parallel and perpendicular component here, is heuristically defined by the averaged orientations of the error ellipses in Fig. 5. These can be computed from the eigenvalues of $\mathrm{D}\{\hat{\mathbf{b}}\}$. Least squares adjustment yields:

$$
\begin{aligned}
& \hat{\mathbf{b}}_{\theta}=\left(\mathbf{T} \overline{\mathbf{A}}_{b}^{T} \mathbf{Q}_{\phi}^{-1} \overline{\mathbf{A}}_{b} \mathbf{T}^{T}\right)^{-1} \mathbf{T} \overline{\mathbf{A}}_{b}^{T} \mathbf{Q}_{\phi}^{-1} \boldsymbol{\phi} \\
& \mathrm{D}\left\{\hat{\mathbf{b}}_{\theta}\right\}=\hat{\sigma}_{0}^{2} \mathbf{Q}_{\theta}=\hat{\sigma}_{0}^{2}\left(\mathbf{T} \overline{\mathbf{A}}_{b}^{T} \mathbf{Q}_{\phi}^{-1} \overline{\mathbf{A}}_{b} \mathbf{T}^{T}\right)^{-1}
\end{aligned}
$$

with:

$\hat{\sigma}_{0}^{2}=\frac{\mathbf{v}_{\phi}^{T} \mathbf{Q}_{\phi}^{-1} \mathbf{v}_{\phi}}{n_{\phi}-u}$, 


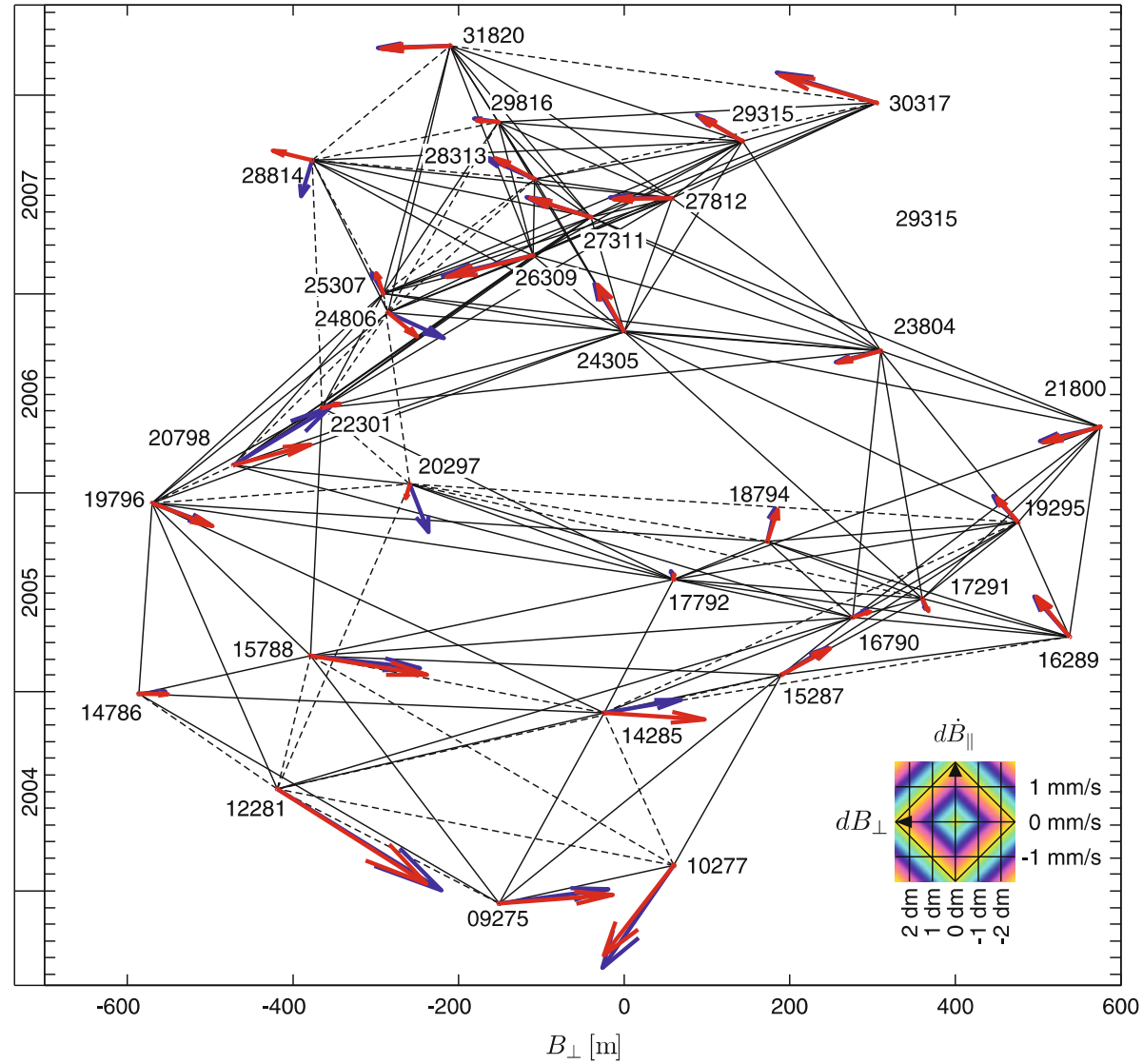

Fig. 6 Network of 163 interferometric combinations of 31 ENVISAT images that has been used to test the estimation of orbit errors. Vertices represent images (acquisitions with indicated orbit numbers), and edges stand for interferograms. The adjusted orbit errors after data snooping are represented by red arrows for the least squares approach and blue arrows for the gridsearch method, respectively. These visualise magnitude and orientation of the fringe gradient, where the number of orbital fringes in the interferogram can be deduced from the legend in the lower

where $u=3$ is the number of unknowns $\left(d \dot{B}_{\|}, d B_{\perp}\right.$ and $\left.\phi_{0}\right)$. $\mathbf{v}_{\phi}$ are the residuals:

$\mathbf{v}_{\phi}=\overline{\mathbf{A}}_{b} \mathbf{T}^{T} \hat{\mathbf{b}}_{\theta}-\boldsymbol{\phi}$.

The selection of an appropriate set of phase observations $\phi$ needs to be addressed. Theoretically, all available pixels could be considered in Eq. (16). However, in many interferograms not every pixel can supply a reliable phase information. In regions of poor coherence, the interferometric phase is practically meaningless and cannot be exploited. On the other hand, it is not advisable to simply exclude poorly coherent pixels below a fixed coherence threshold, since this can lead to inhomogeneous spatial distributions of observations. Thus, local error signals of smaller scale superimposed on the orbital signal can act as leverage observations, dominating the estimates. To avoid such an effect, a homogeneous distribution is enforced by defining a grid on the interferogram and selecting from every grid cell only the pixel with the highest coherence. right corner. The conversion is based on the relation that one fringe in azimuth is equivalent to a baseline error of $d \dot{B}_{\|}=1.7 \mathrm{~mm} / \mathrm{s}$ and one fringe in range corresponds to $d B_{\perp}=26 \mathrm{~cm}$. These factors apply specifically on the test data and account for the actual extent of the scene, which is why they deviate from the numbers in Table 1 . The 31 dashed lines represent interferograms that are identified and rejected as outliers when the gridsearch method is used

For similar reasons, no mutual weighting is applied to the phase observations, constraining $\operatorname{diag}\left(\mathbf{Q}_{\phi}\right) \propto \mathbf{1}$. An inhomogeneous distribution of weights has the same effect as inhomogeneously distributed observations.

\subsection{Baseline error estimation from the wrapped phase}

So far, the presented method estimates baseline errors from unwrapped interferograms only. If reliable unwrapping is cumbersome or even impossible, an alternative gridsearch approach can be pursued. It consists in minimising an objective function of the wrapped phase, incrementally searching the parameter space spanned by $d \dot{B}_{\|}$and $d B_{\perp}$.

From Eq. (16) follows $\mathrm{E}\left\{\boldsymbol{\phi}-\overline{\mathbf{A}}_{b} \mathbf{T}^{T} \mathbf{b}_{\theta}\right\}=\mathbf{0}$. By analogy to the ensemble coherence from Ferretti et al. (2001), a coherence measure is defined as a function of $\mathbf{b}_{\theta}^{T}=\left(d \dot{B}_{\|}, d B_{\perp}\right)$ :

$\gamma\left(\mathbf{b}_{\theta}\right)=\frac{1}{n_{\phi}} \sum_{j=1}^{n_{\phi}} e^{i\left(\phi_{j}^{W}-\overline{\mathbf{a}}_{b, j} \mathbf{T}^{T} \mathbf{b}_{\theta}\right)}$, 
where $\phi^{W}$ is the wrapped interferometric phase and $\overline{\mathbf{a}}_{b, j}$ is the $j$ th row of $\overline{\mathbf{A}}_{b}$. Considering $0 \leq|\gamma| \leq 1$ and $\mathrm{E}\{|\gamma|\}=1$, the estimates $\hat{\mathbf{b}}_{\theta}$ are defined as the set of parameters that maximises $|\gamma|$. Alternatively, $\gamma$ can also be interpreted as a discrete Fourier transform of the two-dimensional signal $e^{i \phi}$ to the $\left(d \dot{B}_{\|}, d B_{\perp}\right)$-domain, where $\hat{\mathbf{b}}_{\theta}$ is constituted of the dominant frequencies. The required computational load is higher than for the least squares method, but still negligible in view of other InSAR processing steps. Whereas the gridsearch approach does not provide any intrinsic quality measures for the estimates, heuristic, peak-to-noise ratio-like indicators can be defined.

A noteworthy drawback of the approach is that the estimates turn out to be unreliable in some cases, in particular when $|\gamma|\left(\mathbf{b}_{\theta}\right)$ has more than one distinct local maximum.

\subsection{Network adjustment}

Given a set of $m$ SAR images, the relative orbit error between two acquisitions $i$ and $j$ can be estimated either directly from interferogram $i j$ or indirectly from some appropriate linear combination of other interferograms. Though mathematically equivalent, both approaches generally do not yield identical results. This is due to individual filter settings for the particular interferograms or data-adaptive filtering. Additionally, different interferometric combinations involve more or less propitious premises for phase unwrapping. Thus, adjusting orbit errors that have been redundantly estimated from linearly dependent interferometric combinations can enhance the precision of the estimates by mitigating "processing noise", even though there is no redundancy in its strict sense.

Furthermore and even more important, this approach provides a mechanism to detect blunders, potentially resulting from unwrapping errors. But even if explicit unwrapping is circumvented by estimating baseline errors directly from the wrapped phase, inconsistencies may occur due to noisy interferograms or unmodelled nuisance signals. As the gridsearch estimator is nonlinear, it is not guaranteed that estimates obtained from equivalent linear combinations of interferograms are identical.

Designing a network with $m$ images, not all $m(m-1)$ possible interferometric combinations are appropriate for orbit error estimation, as the exploitable information degrades with increasing temporal and perpendicular baseline. But even after disregarding too incoherent interferograms, the network design is a trade-off between computational load and reliability. To ensure a good mutual control, every image should be connected to at least three other images. This is a minimum requirement for outlier detection, because an outlier in one out of only two adjacent interferograms cannot be localised. For the following considerations it is further assumed that all images are related to each other by linear combinations of interferograms, i.e., the network design (Fig. 6) can be visualised by a connected graph. If the network falls apart into two or more disjoint parts, each of these parts can be treated separately.

Before adjusting individual baseline errors in a network, it has to be ensured that these parameters refer to a homogeneous reference in several respects. The Frenet frame $\left(\vec{e}_{h}(t)\right.$, $\left.\vec{e}_{a}(t), \vec{e}_{v}(t)\right)$ must be uniformly defined by the orbit of a dedicated, arbitrarily chosen common reference acquisition. This acquisition also defines a uniform time scale $t$ to be applied in Eq. (10). A consistent decomposition of the baseline into parallel and perpendicular component has to be assured by a common mean look angle $\theta_{0}$. Note that it is not required to coregister all images to a single master, since the link between the interferograms is established via the orbits.

The adjustment of misclosures between baseline errors $\mathbf{b}_{\theta, k}^{T}=\left(d \dot{B}_{\|, k}, d B_{\perp, k}\right), k=1 \ldots n$, yields a consistent set of orbit errors $\mathbf{x}_{i}^{T}=\left(d \dot{x}_{\|, i}, d x_{\perp, i}\right), i=1 \ldots m$. If interferogram $k$ is constituted of master acquisition $i$ and slave acquisition $j(i, j \in\{1 \ldots m\})$, the functional model reads $\mathrm{E}\{\mathbf{y}\}=\mathbf{A x}$, or more specifically:

$$
\mathrm{E}\left\{\left(\begin{array}{c}
\vdots \\
\hat{\mathbf{b}}_{\theta, k} \\
\vdots
\end{array}\right)\right\}=\left(\begin{array}{ccccc}
\ldots & \ldots & \ldots & \ldots & \ldots \\
\mathbf{0} & -\mathbf{I}_{2} & \mathbf{0} & \mathbf{I}_{2} & \mathbf{0} \\
\ldots & \ldots & \ldots & \ldots & \ldots
\end{array}\right)\left(\begin{array}{c}
\vdots \\
\mathbf{x}_{i} \\
\vdots \\
\mathbf{x}_{j} \\
\vdots
\end{array}\right)
$$

with $2 n$ "observed" baseline error parameters $\mathbf{y}$, a $2 n \times 2 m$ design matrix A and $2 m$ orbit error parameters $\mathbf{x}$. $\mathbf{I}_{2}$ is a $2 \times 2$ identity matrix. The associated weighting scheme is defined by the stochastic model:

$$
\mathrm{D}\{\mathbf{y}\}=\varsigma_{0}^{2} \mathbf{Q}_{y}=\varsigma_{0}^{2} \operatorname{diag}\left\{\ldots, \hat{\sigma}_{0, k}^{2} \mathbf{Q}_{\theta, k}, \ldots\right\},
$$

subsuming individual covariance matrices from Eq. (19). Note that existing correlations between linearly dependent interferograms are negated here, as their modelling is not straightforward. Contributions of individual interferograms are considered as independent observations.

The estimation of interferogram-specific variance factors $\sigma_{0, k}^{2}$ enables a weighting scheme that allocates higher weights to interferograms whose residual phase pattern closely resembles orbit error signals. Thus, interferograms with strong atmospheric signals that do not match possible orbital error patterns are downweighted, mitigating their influence on the estimates. Such a weighting scheme is not possible if the baseline estimates have been obtained from the wrapped phase as described in Sect. 3.2. In this case, the most evident choice is to assume equal weights for all interferograms and define the mutual weighting of $d \dot{B}_{\|}$and $d B_{\perp}$ by the fringe equivalents from Eq. (5): 
$\mathrm{D}\{\mathbf{y}\}=\varsigma_{0}^{2} \mathbf{I}_{n} \otimes\left(\begin{array}{cc}\left(d \dot{B}_{\|, 2 \pi}\right)^{2} & 0 \\ 0 & \left(d B_{\perp, 2 \pi}\right)^{2}\end{array}\right)$,

where $\otimes$ is the Kronecker product. As the estimation of absolute orbit errors $\mathbf{x}_{k}$ requires a datum definition, two zeromean conditions are introduced:

$$
\sum_{k=1}^{m} \hat{\mathbf{x}}_{k}=\sum_{k=1}^{m}\left(\begin{array}{l}
d \hat{\dot{x}}_{\|, k} \\
d \hat{x}_{\perp, k}
\end{array}\right)=\mathbf{0} .
$$

Whereas there is no physical justification for these conditions, they involve less arbitrary choices than any alternative datum definition. They are expected to yield minimally biased estimates if the number of images $m$ is large and orbit errors are random. As the number of interferograms $n$ is generally larger than $m$, the estimation has $2(n-m+1)$ degrees of freedom and is thus overdetermined. The regularised normal equation system yielding a minimum-norm solution reads:

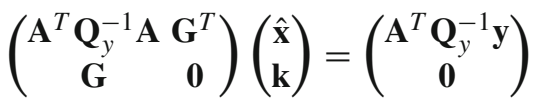

with a $2 \times 2 m$ matrix:

$\mathbf{G}=\left(\begin{array}{llll}\mathbf{I}_{2} & \mathbf{I}_{2} & \cdots & \mathbf{I}_{2}\end{array}\right)$

and Lagrangian multipliers $\mathbf{k}$. Orbit error estimates $\hat{\mathbf{x}}$ are obtained from solving Eq. (27). The corresponding covariance matrix $\hat{\zeta}_{0}^{2} \mathbf{Q}_{\hat{\mathbf{x}} \hat{\mathbf{x}}}$ is a submatrix of:

$\mathrm{D}\left\{\left(\begin{array}{l}\hat{\mathbf{x}} \\ \mathbf{k}\end{array}\right)\right\}=\hat{\zeta}_{0}^{2}\left(\begin{array}{ll}\mathbf{Q}_{\hat{\mathbf{x}} \hat{\mathbf{x}}} & \mathbf{Q}_{\hat{\mathbf{x}} \mathbf{k}} \\ \mathbf{Q}_{\mathbf{k} \hat{\mathbf{x}}} & \mathbf{Q}_{\mathbf{k k}}\end{array}\right)=\hat{\zeta}_{0}^{2}\left(\begin{array}{rr}\mathbf{A}^{T} \mathbf{Q}_{y}^{-1} \mathbf{A} & \mathbf{G}^{T} \\ \mathbf{G} & \mathbf{0}\end{array}\right)^{-1}$

with a variance factor:

$\hat{\zeta}_{0}^{2}=\frac{\mathbf{v}^{T} \mathbf{Q}_{y}^{-1} \mathbf{v}}{2(n-m+1)}$

estimated from the residuals $\mathbf{v}=\mathbf{A} \hat{\mathbf{x}}-\mathbf{y}$. The rescaling by $\hat{\zeta}_{0}^{2}$ accounts only for the processing noise due to which the baseline estimates of equivalent linear combinations of interferograms are not precisely identical.

An equivalent result could alternatively be obtained by means of the pseudo inverse or a singular value decomposition of $\mathbf{A}^{T} \mathbf{Q}_{y}^{-1} \mathbf{A}$. The here proposed approach originates from free adjustment of geodetic networks (Koch 1999) and has its strengths in the enhanced flexibility of datum definition. In some cases it is desirable to consider in Eq. (26) only a subset of acquisitions. For instance, if the network is extended after an initial adjustment by new, recently acquired images, these should be disregarded in Eq. (26) to avoid a systematic shift of all estimates. This can be implemented by zeroing the corresponding coefficients in G (Koch 1999).

The potential of the regularisation approach is illustrated by the following example: For $m=3$ images, the zero-meanconditions read $\sum_{k=1}^{3} \hat{\mathbf{x}}_{k}=\mathbf{0}$, and $\mathbf{G}=\left(\mathbf{I}_{2} \mathbf{I}_{2} \mathbf{I}_{2}\right)$. If a fourth image with a large orbit error is later added to the network and the adjustment is re-performed with zero-mean conditions comprising all four images (i.e., $\sum_{k=1}^{4} \hat{\mathbf{x}}_{k}=\mathbf{0}, \mathbf{G}=$ $\left.\left(\begin{array}{llll}\mathbf{I}_{2} & \mathbf{I}_{2} & \mathbf{I}_{2} & \mathbf{I}_{2}\end{array}\right)\right)$, the orbit error estimates would experience a significant shift also for the initial three images, even if all misclosures are zero. This effect can be avoided by excluding the fourth image from the zero-mean condition: $\sum_{k=1}^{3} \hat{\mathbf{x}}_{k}=\mathbf{0}$.

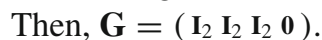

\subsection{Quality control}

In order to detect outliers, Baarda's (1968) data-snooping is applied. The underlying idea of this procedure is to statistically test observations individually on agreement with the mathematical model and to reject outliers iteratively until all tests pass. Baarda's approach requires that the stochastic model of the observations is entirely known. This is not the case in Eqs. (12) and (24), where a fine-tuning by global scaling factors $\sigma_{0}^{2}$ and $\varsigma_{0}^{2}$, respectively, is allowed for. In this context appropriate data-adaptive tests are the $\tau$-test (Pope 1976) or the equivalent $t$-test (Heck 1981; Jäger et al. 2006), following the $\tau$ - or the more common $t$-distribution, respectively. Here, the $t$-test is applied within the framework of iterative data snooping.

There are two levels of data screening. Firstly, it can be tested how well individual unwrapped phase observations match the orbital phase trend. Thus, isolated outliers that are limited to single pixels can be identified and rejected. In the subsequent network adjustment, it can be evaluated if contributions of particular interferograms are biased or not. This second level of data screening is primarily designed to detect unwrapping errors, which often apply to several spatially neighbouring pixels at a time. Then, erroneously unwrapped pixels mask each other, and errors cannot be detected by single outlier statistics on the observation level. Considering further that phase observations are always relative, it is not straightforward for two equally sized regions in an interferogram to determine, which one of the two is unwrapped correctly and which one is not. Quality control on the network level is an effective way to detect these errors and thus one of the basic motivations for the network approach.

Starting on the observation level, the test statistic $T_{\phi, i}$ for the $i$ th phase observation equals its Studentised residual. Assuming normally distributed observations and $\phi_{i}$ being the only outlying phase value, it follows a $t$-distribution (Jäger et al. 2006):

$T_{\phi, i}=\frac{v_{\phi, i}}{\bar{\sigma}_{0} \sqrt{\mathbf{e}_{i}^{T} \mathbf{Q}_{v_{\phi}} \mathbf{e}_{i}}} \sim t_{n_{\phi}-u-1}$,

where $\mathbf{e}_{i}$ is a unit vector of zeros with a one at the $i$ th position. $\mathbf{Q}_{v_{\phi}}$ is the cofactor matrix of the residuals $\mathbf{v}_{\phi}=\left(v_{\phi, i}\right)$ :

$\mathbf{Q}_{\mathbf{v}_{\phi}}=\mathbf{Q}_{\phi}-\overline{\mathbf{A}}_{b} \mathbf{T}^{T} \mathbf{Q}_{\hat{\mathbf{x}} \hat{\mathbf{x}}} \mathbf{T} \overline{\mathbf{A}}_{b}^{T}$. 
Assuming that there is indeed a blunder in the $i$ th observation, the variance factor estimate $\hat{\sigma}_{0}^{2}$ would be biased. Thus, the factor in Eq. (31) is estimated from all residuals except the $i$ th one:

$\bar{\sigma}_{0}^{2}=\frac{\mathbf{v}_{\phi}^{T} \mathbf{Q}_{\phi}^{-1} \mathbf{v}_{\phi}-\frac{\mathbf{v}_{\phi}^{T} \mathbf{Q}_{\phi}^{-1} \mathbf{e}_{i} \mathbf{e}_{i}^{T} \mathbf{Q}_{\phi}^{-1} \mathbf{v}_{\phi}}{\mathbf{e}_{i}^{T} \mathbf{Q}_{\phi}^{-1} \mathbf{Q}_{v_{\phi}} \mathbf{Q}_{\phi}^{-1} \mathbf{e}_{i}}}{n_{\phi}-u-1}$.

If $T_{\phi, i}$ exceeds a dedicated threshold deduced from the $t$-distribution, the $i$ th observation is rejected, and the parameters are re-estimated from the remaining ones. This procedure is repeated until all test statistics fall below the threshold. It has to be taken care that this iterative rejection does not yield an unbalanced spatial distribution of observations, which could entail leverage effects as addressed in Sect. 3.1. Consequently, only a limited number of observations should be rejected. Possibly, robust estimation approaches (Koch 1999) would be more effective in this case to find a more appropriate balance between quality and spatial distribution of the observations. However, a successful application of these concepts would require further and thorough research to ensure a reliable result.

Outlier detection on the network level follows the same scheme, testing the contribution of every interferogram $k$ individually. The alternative hypothesis reads:

$\mathrm{E}\left\{\mathbf{b}_{\theta, k}\right\}=\mathbf{A}_{k} \mathbf{x}+\nabla_{k}$,

where $\mathbf{A}_{k}$ is the line-block of $\mathbf{A}$ corresponding to interferogram $k$ and $\nabla_{k}^{T}=\left(\nabla \dot{B}_{\|, k}, \nabla B_{\perp, k}\right)$ are two nuisance parameters, modelling the assumed bias in terms of baseline errors. Provided that interferogram $k$ is the only interferogram that contributes erroneous baseline error estimates to the network, the best estimate for $\nabla_{k}$ is (Jäger et al. 2006):

$\hat{\boldsymbol{\nabla}}_{k}=-\left(\mathbf{Q}_{y, k}^{-1}-\mathbf{Q}_{y, k}^{-1} \mathbf{A}_{k} \mathbf{Q}_{\hat{\mathbf{x}} \hat{\mathbf{x}}} \mathbf{A}_{k}^{T} \mathbf{Q}_{y, k}^{-1}\right)^{-1} \mathbf{Q}_{y, k}^{-1} \mathbf{v}_{k}$

where $\mathbf{v}_{k}=\mathbf{A}_{k} \hat{\mathbf{x}}-\hat{\mathbf{b}}_{\theta, k}$ comprises the two elements of $\mathbf{v}$ that concern interferogram $k$. The significance of $\hat{\nabla}_{k}$ can be evaluated by a generalised t-test for two parameters (Jäger et al. 2006):

$T_{B, k}=-\frac{\mathbf{v}_{k}^{T} \mathbf{Q}_{y, k}^{-1} \hat{\nabla}_{k}}{2 \bar{\zeta}_{0}^{2}} \sim F_{2,2(n-m)}$

with:

$\bar{\zeta}_{0}^{2}=\frac{\mathbf{v}^{T} \mathbf{Q}_{y}^{-1} \mathbf{v}+\mathbf{v}_{k}^{T} \mathbf{Q}_{y, k}^{-1} \hat{\nabla}_{k}}{2(n-m)}$.

If the highest $T_{B}$ exceeds a dedicated threshold, this time deduced from the Fisher distribution, it can be checked in the first place if there is an unwrapping error in interferogram $k$ that can be corrected manually. Otherwise, its contribution is rejected, and the procedure is repeated until all test statistics fall below the threshold. Rejection must not be pursued too extensively, guaranteeing that the contribution of every interferogram is controlled by at least one linear combination of other interferograms in the network.

\section{Application}

The proposed approach for estimating orbit errors has been tested on a set of 31 ENVISAT acquisitions from a scene in Western Australia (track 203, frame 4221) between December 2003 and April 2008. The region has a semi-arid climate, the land use being dominated by dryland cropping and some salt lakes. These conditions go along with a good interferometric coherence, which was the reason to choose this test area. A network of 163 interferograms has been set up with a maximum perpendicular baseline of $743 \mathrm{~m}$ and a maximum temporal baseline of 560 days (see Fig. 6). It was aimed to include as many interferograms as possible, the only requirement being that unwrapping is reliably feasible. Three sample interferograms are pictured in Fig. 7.

InSAR processing has been performed with the Delft Object-Oriented Radar Interferometric Software DORIS (Kampes et al. 2004) using precise orbits from the French Centre National d'Etudes Spatiales (CNES). Topographic height variations, which are below $200 \mathrm{~m}$, have been accounted for with a 3"-DEM product from the Shuttle Radar Topography Mission (SRTM). To maximise coherence, all interferograms have been multilooked by a factor 25 in azimuth and 5 in range, yielding pixels of approximately $100 \times 100 \mathrm{~m}^{2}$ size. Adaptive phase filtering (Goldstein and Werner 1998) has been applied to facilitate unwrapping, which has been carried out using the Statistical-Cost Network-Flow Algorithm for Phase Unwrapping (SNAPHU; Chen and Zebker 2001). Subdividing the interferograms into 1260 tiles of $30 \times 30$ pixels, only the most coherent pixel from each tile contributes to the estimation to guarantee a spatially homogeneous distribution of observations. A small number of tiles has been disregarded due to lack of pixels with a coherence estimate above 0.25 .

\subsection{Stochastic modelling}

The choice of an appropriate covariance matrix for the phase observations $\phi_{i}$ in Eq. (12) has several implications: It defines the mutual weighting between the contributions of individual pixels, it directly affects the quality measures, i.e., standard deviations, of the estimated orbit errors and it is an important prerequisite for outlier detection. As orbit errors are the only effect that is considered in the functional model of Eq. (16), all other contributions like deformation, 


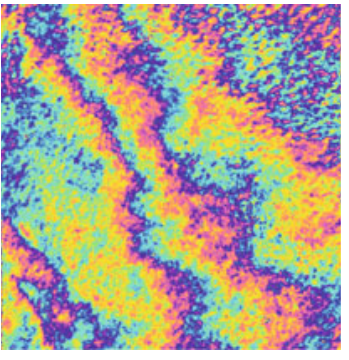

09275-10277

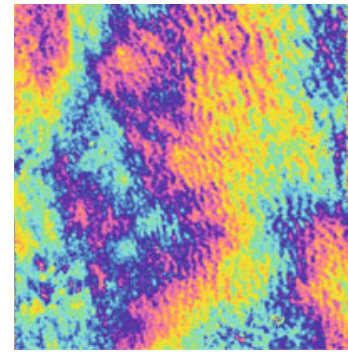

$14786-15788$

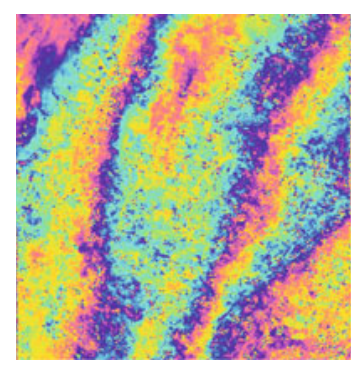

$22301-24806$
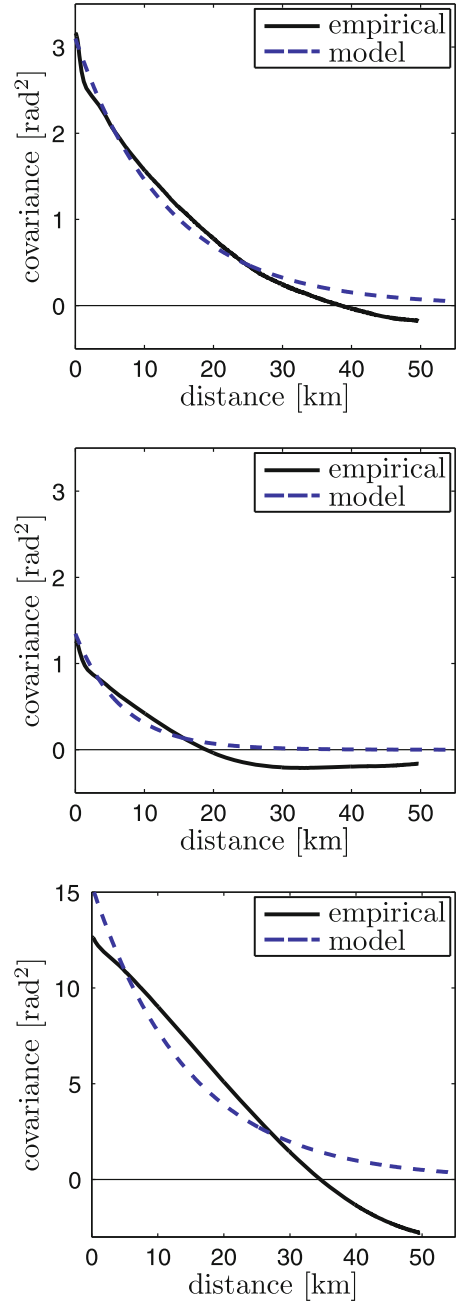

Fig. 7 Sample interferograms and their associated covariance functions. The interferograms covering the whole scene of $100 \times 100 \mathrm{~km}^{2}$ have already been corrected for reference phase and topographic phase. The empirical covariance functions have been fitted by the double exponential model $C_{e}(r)$ from Eq. (39)

atmosphere and noise are absorbed in the stochastic model and would thus have to be accounted for.

This requirement is in conflict with a number of constraints. Allowing the phase variances to account for decorrelation noise as a function of coherence would allocate different weights to the individual pixels. This has to be avoided to prevent leverage effects that could result from an inhomogeneous spatial distribution of weights. Admitting thus only models with homogeneous variances, the only matter to be settled is the question if correlations should be assumed or not. As the atmospheric propagation delay definitely is a spatially correlated effect, it suggests itself not to ignore this stochastic behaviour. However, the characteristic of the associated covariances is very different for individual interferograms (Hanssen 2001). The consequential need to individually tailor covariance models is opposed to the requirement of a generally applicable methodology. Hence, the covariance of two pixels is assumed to be an isotropic function of their spatial distance $r$, for which the most simplistic choice would be:

$C_{0}(r ; c)=\left\{\begin{array}{ll}c, & r=0 \\ 0, & r>0\end{array}\right.$,

where $c>0$. This model, implying uncorrelated observations, does not even require adaption, because the parameter $c$ is arbitrary due to the a posteriori estimation of $\hat{\sigma}_{0}^{2}$ in Eq. (20). But it does not reflect realistic conditions either. If a more sophisticated model is supposed to be applied, a two-dimensional covariance function can be estimated from the power spectrum of an interferogram by application of an inverse Fourier transformation (Hanssen 2001). This requires second order stationarity of the underlying stochastic process, of which the interferogram is a realisation in this context. To fulfil this requirement, the assumed contribution of orbital errors can be removed from the interferograms by subtraction of a linear trend, which is a sufficiently good approximation for this purpose.

To avoid a bias due to decorrelation noise, the power spectrum is low-pass filtered beforehand. Circular averaging finally yields a one-dimensional covariance function. As the thus obtained function is only defined for spatial wavelengths below half the size of the interferogram, it is extrapolated by fitting a double exponential analytical model:

$C_{e}\left(r ; c_{1}, c_{2}, a\right)=c_{1} e^{-\frac{r}{1 k m}}+c_{2} e^{-\frac{r}{a}}$.

This model has been chosen, because it matches well the empirical covariance functions and more sophisticated, better-fitting models do not qualify by a better performance. The empirical choice of $1 \mathrm{~km}$ as correlation length of the first exponential function can be motivated with the transition between different atmospheric scaling regimes at $2 \mathrm{~km}$ distance (Hanssen 2001). $\left(e^{-r /(1 \mathrm{~km})}\right.$ has decayed by $90 \%$ at $r=2 \mathrm{~km}$.)

There is still a number of deficiencies left. As the spectral method does not account for wavelengths that exceed the extent of the interferogram, the resulting covariance functions are significantly biased for about 20 interferograms containing distinct nonlinear atmospheric trends (e.g., ifg. 22301-24806, see Fig. 7). Some interferograms (like 1478615788 in Fig. 7) expose distinct anisotropic features, which have been disregarded in the modelling to keep the approach simple.

The factual benefit of the double exponential covariance model $C_{e}(r)$ compared to the simpler model without covariances $C_{0}(r)$ will be evaluated in the following. 
Table 3 Results of network adjustment for the least squares (lsq.) approach using the covariance functions $C_{0}(r)$ or $C_{e}(r)$, respectively, and the gridsearch method

\begin{tabular}{|c|c|c|c|c|c|c|c|c|c|c|}
\hline & $d \hat{\dot{B}}_{\|}(\mathrm{mm} / \mathrm{s})$ & $d \hat{B}_{\perp}(\mathrm{cm})$ & $d \hat{B}_{\mathrm{fr}}(\mathrm{fr})$. & $d \hat{\dot{x}}_{\|}(\mathrm{mm} / \mathrm{s})$ & $d \hat{x}_{\perp}(\mathrm{cm})$ & $d \hat{x}_{\mathrm{fr}}(\mathrm{fr})$. & $v_{\dot{B}_{\|}}(\mathrm{mm} / \mathrm{s})$ & $v_{B_{\perp}}(\mathrm{cm})$ & $v_{B_{\mathrm{fr}}}(\mathrm{fr}$.) & $\hat{\nabla} B_{\mathrm{fr}}(\mathrm{fr})$. \\
\hline \multicolumn{11}{|c|}{ 1sq. $C_{0}(r)$} \\
\hline med & 0.64 & 16.3 & 1.11 & 0.55 & 18.6 & 1.12 & 0.03 & 0.3 & 0.02 & \\
\hline $\max$ & 3.43 & 94.8 & 4.78 & 2.68 & 65.2 & 4.08 & 0.30 & 2.0 & 0.22 & 0.26 \\
\hline$\hat{\sigma}_{\min }$ & 0.01 & 0.2 & 0.01 & 0.01 & 0.1 & 0.01 & & & & \\
\hline$\hat{\sigma}_{\max }$ & 0.12 & 1.8 & 0.10 & 0.03 & 0.4 & 0.02 & & & & \\
\hline \multicolumn{11}{|c|}{ lsq. $C_{e}(r)$} \\
\hline med & 0.65 & 17.1 & 1.02 & 0.50 & 18.9 & 1.10 & 0.04 & 0.4 & 0.04 & \\
\hline $\max$ & 3.12 & 91.6 & 4.43 & 2.31 & 65.0 & 3.83 & 0.47 & 3.5 & 0.33 & 0.35 \\
\hline$\hat{\sigma}_{\min }$ & 0.01 & 0.1 & 0.01 & 0.01 & 0.2 & 0.01 & & & & \\
\hline$\hat{\sigma}_{\max }$ & 0.16 & 2.3 & 0.13 & 0.06 & 0.8 & 0.05 & & & & \\
\hline \multicolumn{11}{|c|}{ gridsearch } \\
\hline med & 0.76 & 18.9 & 1.23 & 0.58 & 19.9 & 1.10 & 0.13 & 3.1 & 0.27 & \\
\hline $\max$ & 5.59 & 120.2 & 7.16 & 3.93 & 72.2 & 4.09 & 3.84 & 88.1 & 5.64 & 6.72 \\
\hline$\hat{\sigma}_{\min }$ & 0.82 & 12.5 & 0.68 & 0.22 & 3.3 & 0.18 & & & & \\
\hline$\hat{\sigma}_{\max }$ & 0.82 & 12.5 & 0.68 & 0.41 & 6.2 & 0.34 & & & & \\
\hline \multicolumn{11}{|c|}{$\begin{array}{l}\text { gridsearch } \\
\& \text { data snooping }\end{array}$} \\
\hline med & 0.68 & 17.3 & 1.16 & 0.60 & 20.2 & 1.24 & 0.04 & 0.5 & 0.04 & \\
\hline $\max$ & 3.77 & 81.9 & 4.97 & 2.97 & 71.6 & 4.48 & 0.19 & 2.7 & 0.16 & 0.25 \\
\hline$\hat{\sigma}_{\min }$ & 0.07 & 1.1 & 0.06 & 0.02 & 0.3 & 0.02 & & & & \\
\hline$\hat{\sigma}_{\max }$ & 0.07 & 1.1 & 0.06 & 0.05 & 0.8 & 0.05 & & & & \\
\hline
\end{tabular}

Given are the median and the maximum of the absolute values of observed baseline errors $d \hat{B}$, adjusted orbit errors $d \hat{x}$, residuals $v_{B}$ and estimated biases $\hat{\nabla} B$ as well as their minimum and maximum standard deviations. $d \hat{B}_{\mathrm{fr}}$ combines the effects in $d \hat{\dot{B}}_{\|}$and $d \hat{B}_{\perp}$ by converting them to fringes (fr.) following Eq. (40). $d \hat{x}_{\mathrm{fr}}, v_{B_{\mathrm{fr}}}$ and $\hat{\nabla} B_{\mathrm{fr}}$ have analogous meanings

\subsection{Performance}

As the true orbit errors are unknown, it is not feasible to evaluate the actual accuracy of their estimates. Nevertheless, the parameters' plausibility can be checked roughly, and their mutual consistency can be inferred from misclosures in the network. Whereas least squares and gridsearch estimates can easily be compared with each other, it is not straightforward to assess the innovation of both estimators with respect to the common approach of removing linear ramps. A direct numerical comparison fails due to the different parameterisations and their effect on the phase.

Table 3 summarises the adjustment results. Assuming uncorrelated observations $\left(C_{0}(r)\right)$, baseline errors up to $d \dot{B}_{\|}=3.4 \mathrm{~mm} / \mathrm{s}(2.0$ fringes $)$ and $d B_{\perp}=95 \mathrm{~cm}(3.6$ fringes) have been estimated by the least squares (lsq.) method. These dimensions are hardly explainable by orbit errors and suggest that large-scale variations of the atmospheric delay leak into the baseline error estimates. This is plausible, as the nonlinear signal in interferogram 2230124806 proves that gradients in the atmospheric propagation delay of two fringes over half a scene $(50 \mathrm{~km})$ are possible (see Fig. 7). The network adjustment yields absolute orbit errors up to $2.7 \mathrm{~mm} / \mathrm{s}$ in $\dot{x}_{\|}$(1.6 fringes in azimuth) and $65 \mathrm{~cm}$ in $x_{\perp}$ ( 2.5 fringes in range). The maximum residuals of $0.3 \mathrm{~mm} / \mathrm{s}$ in $d \dot{B}_{\|}$and $2 \mathrm{~cm}$ in $B_{\perp}$ are much smaller than in Bähr and Hanssen (2010), where the same dataset has been analysed with a similar network design. This can be explained by a range timing error in the annotations of image 10277 of $0.3 \mu$ s (12 pixels) that could be reliably identified and corrected.

In Table 3, baseline errors (and analogously orbit errors, residuals and estimated biases) are also quantified in terms of the total number of fringes that they induce into an interferogram:

$d B_{\mathrm{fr}}:=\left|\frac{d \dot{B}_{\|}}{d \dot{B}_{\|, 2 \pi}}\right|+\left|\frac{d B_{\perp}}{d B_{\perp, 2 \pi}}\right|$.

The conversion to fringes follows Eq. (5), where $d \dot{B}_{\|, 2 \pi}=$ $1.7 \mathrm{~mm} / \mathrm{s}$ and $d B_{\perp, 2 \pi}=26 \mathrm{~cm}$ are applicable for the data at hand. An analogous conversion is applied to the mutual 
Table 4 Comparison of orbit error estimates obtained by the least squares (1sq.) approach, using the covariance models $C_{0}(r)$ or $C_{e}(r)$, respectively, and the gridsearch method

\begin{tabular}{|c|c|c|c|c|c|c|}
\hline & \multicolumn{3}{|c|}{ Without data snooping } & \multicolumn{3}{|c|}{ With data snooping } \\
\hline & $\begin{array}{l}\text { lsq. } \\
C_{0}(r)\end{array}$ & $\begin{array}{l}\text { lsq. } \\
C_{e}(r)\end{array}$ & $\begin{array}{l}\text { grid- } \\
\text { search }\end{array}$ & $\begin{array}{c}\text { lsq. } \\
C_{0}(r)\end{array}$ & $\begin{array}{l}\text { lsq. } \\
C_{e}(r)\end{array}$ & $\begin{array}{l}\text { grid- } \\
\text { search }\end{array}$ \\
\hline $\begin{array}{l}\text { Rejected interferograms } \\
\text { med/max deviation (fringes) } \\
\text { without data snooping }\end{array}$ & 0 & 0 & 0 & 2 & 1 & 31 \\
\hline lsq., $C_{0}(r)$ & & $0.05 / 0.39$ & $0.19 / 0.91$ & $0.00 / 0.02$ & $0.05 / 0.39$ & $0.09 / 1.25$ \\
\hline lsq., $C_{e}(r)$ & & & $0.20 / 1.15$ & $0.05 / 0.40$ & $0.00 / 0.02$ & $0.14 / 1.30$ \\
\hline gridsearch & & & & $0.19 / 0.92$ & $0.20 / 1.16$ & $0.12 / 1.44$ \\
\hline $\begin{array}{l}\text { with data snooping } \\
\text { lsq., } C_{0}(r)\end{array}$ & & & & & $0.05 / 0.40$ & $0.09 / 1.25$ \\
\hline lsq., $C_{e}(r)$ & & & & & & $0.13 / 1.31$ \\
\hline
\end{tabular}

All three estimators have been evaluated with and without application of data snooping, for which the number of rejected interferograms is indicated in each case. For every pairing of approaches, both the maximum and the median deviation between the estimated orbit error parameters is given, converted to fringes by analogy to Eq. (40): $d \hat{x}_{\mathrm{fr}}=\left|d \hat{\dot{x}}_{\|} / d \dot{B}_{\|, 2 \pi}\right|+\left|d \hat{x}_{\perp} / d B_{\perp, 2 \pi}\right|$

deviations of different approaches in Table 4. The comparison there reveals that the choice of the covariance function has only little effect on the estimates, the median deviation being 0.05 fringes. For a few acquisitions, the estimated orbit errors differ significantly, showing deviations up to 0.40 fringes. Hence, it can be concluded that in spite of the good precision (i.e., standard deviation) of the estimated orbit errors better than 0.05 fringes, the factual accuracy may be at the level of a few tenths of fringes in some cases.

The estimated standard deviations are throughout smaller if uncorrelated observations $\left(C_{0}(r)\right)$ are assumed, whereas the quality indicators from correlated observations $\left(C_{e}(r)\right)$ are probably more realistic. Although the double exponential covariance model $C_{e}(r)$ is still a crude approximation of the true stochastic behaviour of the interferometric phase with some unresolved deficiencies, it is supposed to be a closer approximation of reality than the simple model $C_{0}(r)$.

As all interferograms have been processed with care, the presence of unwrapping errors in the dataset can basically be excluded. However, regardless the choice of the covariance function, there are interferograms that do not pass the outlier test in Eq. (36) at a significance level of $\alpha=0.001$. This is probably due to remaining deficiencies of the stochastic model addressed in Sect. 4.1. If data snooping is applied, not more than two interferograms are rejected before all tests pass while the change of the estimated orbit errors is below 0.02 fringes and thus negligible (see Table 4). Hence, data snooping cannot be considered useless, since the contributions of the majority of interferograms are accepted. Its capability to detect unwrapping errors will be analysed in detail in the next subsection.

Considering the least squares solution as an unbiased reference, the estimates obtained by the gridsearch method are distinctly unreliable in some cases. Figure 8 a shows that there are high deviations of up to 5.7 fringes. These occur frequently in the presence of a nonlinear large-scale atmospheric signal and go along with the presence of more than one distinct local maximum in the search space (see Fig. 8b). Hence, the ratio between the highest local maximum $\gamma_{1}$ and the second-highest local maximum $\gamma_{2}$ can be considered an indicator for the reliability of the estimation. From Fig. 8a can be seen that the probability of a biased estimate is high if $\gamma_{1} / \gamma_{2}<1.5$.

The internal consistency of the estimates obtained by the gridsearch method is poor, which is suggested by the high residuals in Table 3. Applying data snooping with a significance level of $\alpha=0.001$, as many as 31 interferograms are rejected before all tests pass. With standard deviations of orbit errors below 0.05 fringes, the consistency of the revised network is of a similar quality as the least squares solution (see Table 3). However, the estimates for some acquisitions deviate on the one-fringe level (see Fig. 6 and Table 5), which can be explained by non-equivalent objective functions of the two approaches. Hence, biased contributions of particular interferograms estimated by the gridsearch method cannot be reliably identified by data snooping.

\subsection{Detectability of outliers}

Two statistical tests have been proposed to detect unwrapping errors. On the observation level, $T_{\phi}$ indicates if individual phase observations deviate significantly from the estimated orbital error signal. On the network level, largescale unwrapping errors can be detected with $T_{B}$. In the following it is evaluated for both tests, if the associated statistical distributions do indeed apply to the test statistics in context of 


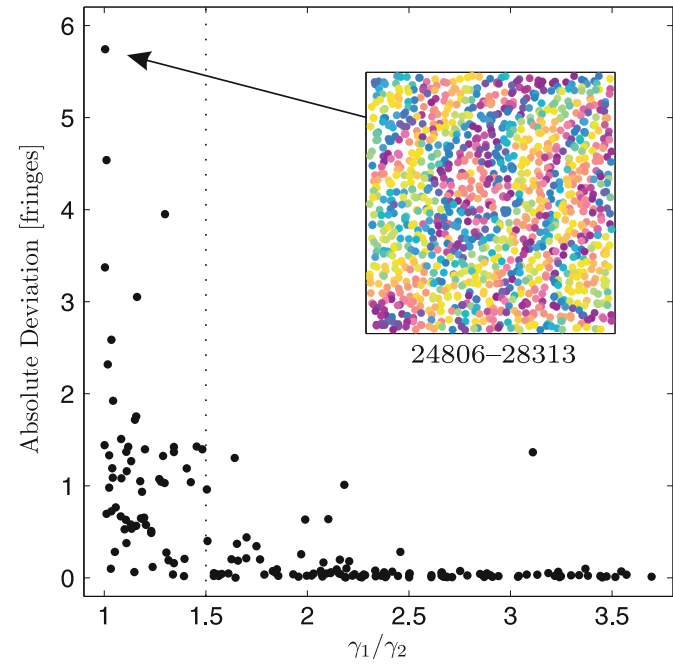

(a) Deviation of gridsearch from least squares estimates

Fig. 8 a Absolute deviations of the baseline error parameters obtained by the girdsearch method from the least squares estimates (from uncorrelated observations). By analogy to Eq. (40), the deviations in the respective components have been normalised to their fringe equivalent and subsequently summed up per interferogram. They are plotted as a function of the ratio $\gamma_{1} / \gamma 2$ between the highest and the second-highest local maximum in the search space $|\gamma|\left(d \dot{B}_{\|}, d B_{\perp}\right)$. The highest deviation has been observed in interferogram 2480628313, the observations from which are visualised in the

the chosen stochastic model. Subsequently, the performance in detecting simulated unwrapping errors is tested.

The applicability of the respective statistical distributions in Eqs. (31) and (36) is evaluated by Pearson's chi-square goodness-of-fit test (Kreyszig 1979). Doing so, the test statistics are binned into $N=15$ intervals. Then, the number of tests $h_{i}$ in each interval is compared to the theoretical number of tests $h_{i, 0}$ that is supposed to be in that interval if the associated statistical distribution applies. Finally, the goodness-of-fit test is based on the difference of these numbers:

$T_{\chi^{2}}=\sum_{i=1}^{N} \frac{\left(h_{i}-h_{i, 0}\right)^{2}}{h_{i, 0}} \sim \chi_{N-1}^{2}$.

To evaluate the test $T_{\phi}$ on the observation level, $T_{\chi^{2}}$ has been computed for all 163 interferograms after iteratively rejecting a small number of outliers. This is necessary to guarantee that the samples are not contaminated by blunders. Even for a small level of significance $\alpha=0.1 \%$, the $t$-distribution can be validated for only $73 \%$ of the interferograms if uncorrelated observations are assumed $\left(C_{0}(r)\right)$. For the double exponential model $C_{e}(r)$, the validation succeeds for only $22 \%$. These results indicate that both models are incapable of adequately describing the stochastic behaviour of the observations for the general case. Nevertheless, the performance of this test is only of secondary importance if only a limited number of distinct blunders is rejected. Mod-

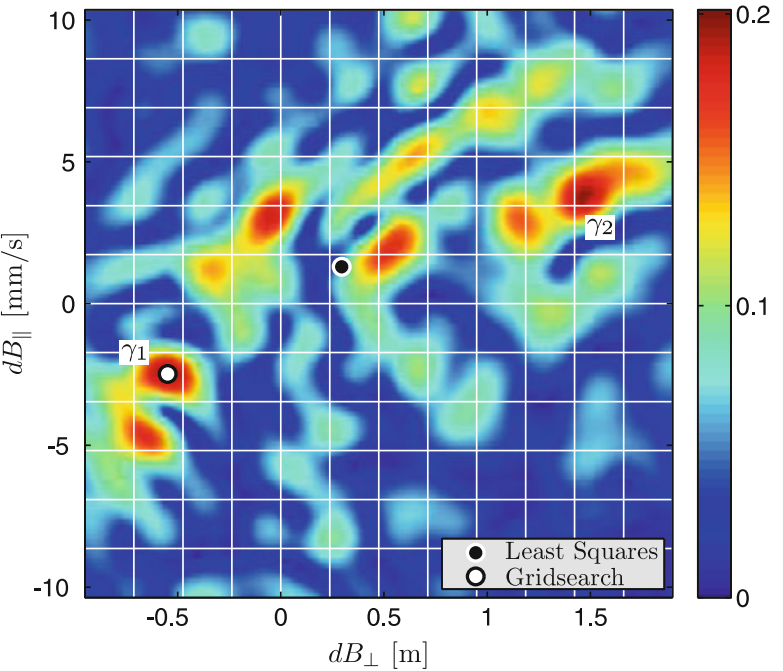

(b) $|\gamma|\left(d \dot{B}_{\|}, d B_{\perp}\right)$ for interferogram 24806-28313

subframe. b Coherence measure $|\gamma|$ of interferogram 2480628313 computed for incrementally varied baseline error values $\left(d \dot{B}_{\|}, d B_{\perp}\right)$. The interval between the white grid lines corresponds to one fringe according to Eq. (5). The gridsearchsolution is defined by the highest value of $|\gamma|$, from which the least squares solution has a distance of 5.7 fringes-2.2 in azimuth $\left(d \dot{B}_{\|}\right)$ and 3.6 in range $\left(d B_{\perp}\right)$. Note that the least squares solution does not necessarily coincide with a local maximum in the search space, which is due to the differing objective functions of the two estimators

erate biases in a small number of pixels do not distort the orbit error estimates significantly due to the high number of observations. More crucial is the test $T_{B}$ on the network level, for which the goodness-of-fit test passes for any significance level below $9 \%\left(C_{0}(r)\right)$ or $11 \%\left(C_{e}(r)\right)$, respectively. This is a promising result, confirming the validity of this test.

To assess the sensitivity of $T_{B}$ with respect to large-scale unwrapping errors, such errors have been simulated as demonstrated in Fig. 9. 163 case studies have been carried out, in each of which one of the 163 interferograms is contaminated by unwrapping errors with incrementally varied magnitudes. The respective numbers of case studies, in which an unwrapping error of a specified magnitude is detected are listed in Table 6. It turns out that the more sophisticated covariance model $C_{e}(r)$ promotes the detectability of outliers, as the success rate is significantly higher for errors below 0.3 fringes compared to $C_{0}(r)$. The finding that errors above 0.5 fringes still remain undetected appears troubling but is relativised by the circumstance that the interferograms in question are associated with small weights in the network, mitigating their influence on the estimates.

Recapitulatory can be stated that the effort of adapting a stochastic model $C_{e}(r)$ with correlated observations has indeed some benefit in the detection of outliers, even though it is not rigorously adequate in all respects. However, the performance of the model $C_{0}(r)$ with uncorrelated observations is acceptable. 
Table 5 Results of reprocessing with corrected orbits for four sample interferograms

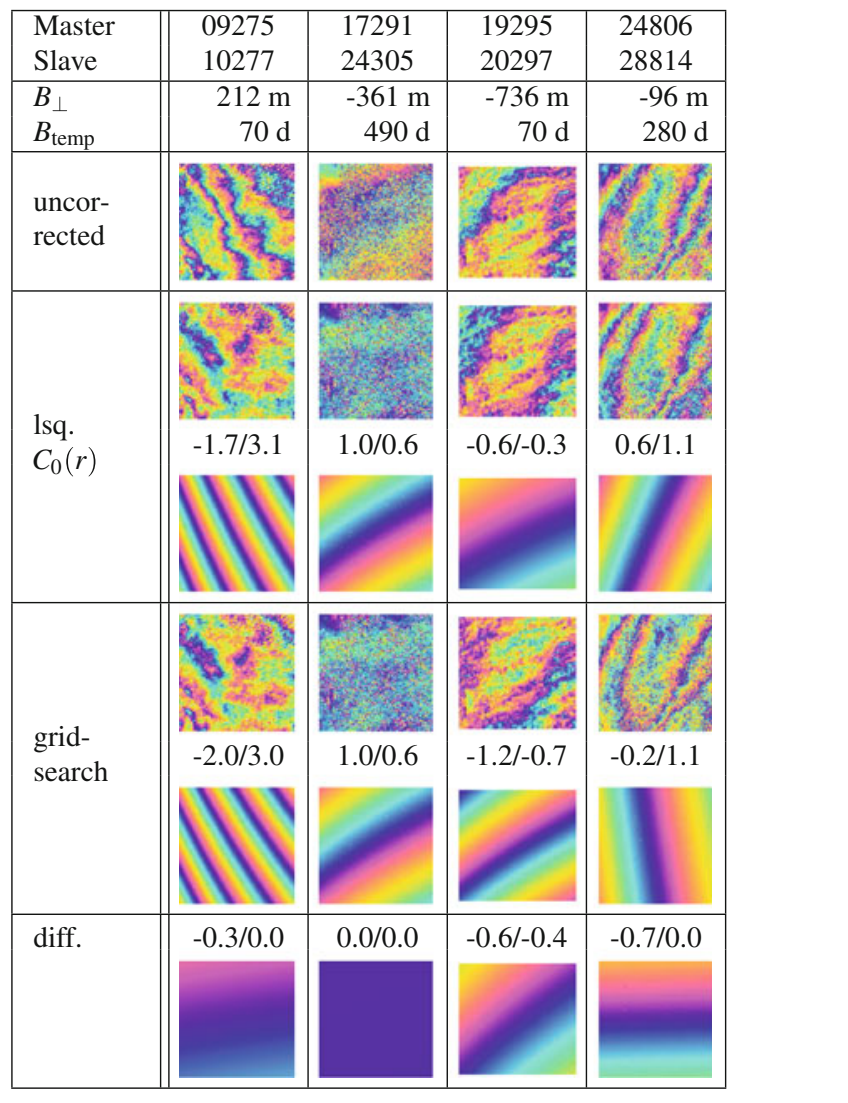

For the least squares (lsq.) and the gridsearch approach, the corrected interferogram and the difference with respect to the uncorrected interferogram are pictured. The corrections have been estimated by network adjustment after data snooping, assuming uncorrelated observations $\left(C_{0}(r)\right)$ in case of the least squares estimator. They are given here in fringes in azimuth and fringes in range, respectively, following Eq. (5). The last row shows the difference between the two approaches. Whereas the estimates for the orbital error signals seem reasonable for both approaches, the results differ for a small number of interferograms. Note that for the great majority of interferograms the difference is insignificant, as it is the case for 17291-24305 (see Fig. 6)
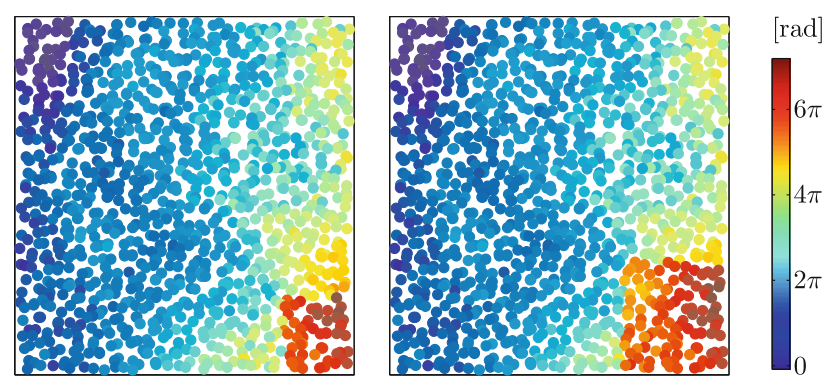

Fig. 9 Simulation of unwrapping errors, exemplarily demonstrated on interferogram 14786-15788. All phase observations in a quadratically confined area in the lower right corner are shifted by $2 \pi$. The fringe equivalent of the induced error signal according to Eq. (40) is 0.4 fringes for the left and 0.8 fringes for the right example
Table 6 Number of interferograms that do not pass the outlier test $T_{B}$ if they are contaminated by a simulated unwrapping error as demonstrated in Fig. 9

\begin{tabular}{lcr}
\hline Simulated error (fringes) & \multicolumn{2}{l}{ Covariance model } \\
\cline { 2 - 3 } & $C_{0}(r)$ & $C_{e}(r)$ \\
\hline 0.05 & $2(1 \%)$ & $42(26 \%)$ \\
0.10 & $3(2 \%)$ & $85(52 \%)$ \\
0.15 & $27(17 \%)$ & $109(67 \%)$ \\
0.20 & $67(41 \%)$ & $119(73 \%)$ \\
0.25 & $107(66 \%)$ & $129(79 \%)$ \\
0.30 & $135(83 \%)$ & $141(87 \%)$ \\
0.40 & $146(90 \%)$ & $149(91 \%)$ \\
0.50 & $156(96 \%)$ & $156(96 \%)$ \\
0.60 & $162(99 \%)$ & $160(98 \%)$ \\
0.70 & $163(100 \%)$ & $162(99 \%)$ \\
0.80 & $163(100 \%)$ & $163(100 \%)$ \\
\hline
\end{tabular}

\subsection{Sequential versus comprehensive adjustment}

The organisation of the adjustment in two steps, firstly estimating individual baseline parameters to be adjusted subsequently in the network, makes the procedure conveniently modularisable and reproducible. A more rigorous approach would be a comprehensive formulation of the functional relationship, i.e.,

$$
\mathrm{E}\left\{\left(\ldots, \boldsymbol{\phi}_{k}^{T}, \ldots\right)^{T}\right\}=f(\mathbf{x}),
$$

involving an adjustment in only one step. Thus, it could be accounted for different look directions $\vec{r}_{M}$ and $\vec{r}_{S}$ of master and slave, respectively, as it has been proposed by Kohlhase et al. (2003). For the two-step approach, they are simply averaged in Eq. (10). But as the look directions are almost collinear in spaceborne SAR, the bias due to averaging is small enough to be negligible.

A second advantage of a comprehensive approach would be that the mutual weighting of the contributions of different interferograms by $\hat{\sigma}_{0, k}^{2}$ and the estimation of a global variance level $\hat{\zeta}_{0}^{2}$ could be unbiasedly performed in one step by applying variance component estimation (Koch 1999). The benefit has been evaluated, revealing no significant change in the estimates. Whereas the resulting covariance information differs significantly, it does not improve the performance in outlier detection.

\section{Separability of signal components}

The proposed method is based on the assumption that besides orbit errors there are no other systematic components in the residual interferometric phase, which is generally not true. 
Consequently, unmodelled contributions of ground deformation or atmospheric propagation delay may leak into the estimates, and the subsequent elimination of orbital errors can bias the respective signal of interest. This mechanism is unavoidable, but it can be mitigated by exploiting the spatiotemporal characteristic of the orbital error signal. This signal always affects a radar scene as a whole and changes arbitrarily from one acquisition to the next, revealing a large spatial wavelength and an uncorrelated temporal behaviour.

Compared to the revisit intervals of SAR satellites, the atmospheric state changes fast enough to consider its influence on subsequent acquisitions as completely uncorrelated. In the spatial domain, the spectrum of effects ranges from turbulent mixing on small scales over vertical stratification due to topographic variations to large-scale gradients of temperature, pressure or ionospheric electron content. The turbulent component remains practically unaffected by an orbit error correction due to its differing spatial characteristics, and the effect of stratification can eventually be captured by correlation with the topographic height. Only phase gradients reflecting large-scale weather patterns are not separable from orbital effects without complementary measurements or weather models. However, if not propagation delay but deformation is the dedicated signal of interest, it is acceptable if part of the atmospheric contribution is mistaken as orbit error.

As to deformation phenomena, it can be stated that localised signals remain basically unaffected by the orbit error correction. This does not apply to large-scale ground movements, where the separation from orbit errors is not possible without additional measurements or assumptions. If available, ground velocities can be constrained at selected points by independent geodetic measurements (Lundgren et al. 2009). Otherwise, the temporal correlation of deformation can be exploited, which is a distinction compared to orbit errors and atmospheric effects (Ferretti et al. 2001; Hooper et al. 2007). However, this self-evident assumption has proven invalid in some cases where temporally correlated spatial trends have been observed in InSAR time series that cannot be explained by deformation (Hooper et al. 2007; Ketelaar 2009).

If temporal correlation properties are nevertheless supposed to be exploited to mitigate the bias of deformation estimates, it suggests itself to high-pass filter the error estimates before correcting the orbits and subsequently analysing deformation. Thus, no prior assumptions have to be made on the spatial characteristic of the deformation signal, since the temporal filtering is performed in the very domain in which the potential contamination takes place. Of course, a most appropriate approach would be the joint estimation of orbit errors and deformation in an all-comprehensive model, which is beyond the scope of this work.
Another conceivable application scenario for the proposed method involves Permanent or Persistent Scatterer (PS) approaches, for which a number of different processing chains have been developed (Ferretti et al. 2001; Hooper et al. 2007; Ketelaar 2009). These are designed to deduce deformation estimates for temporally stable point scatterers and generally involve an initial estimate of orbit errors to support an optimal identification of PS. At this stage, the here proposed method could get involved. Additionally, the bias due to deformation could be mitigated by subtracting the estimated deformation signal from the original interferograms and iteratively re-estimating orbit errors.

\section{Conclusions}

A reliable method to improve orbital state vectors has been proposed and evaluated. It involves estimation of relative orbit errors, i.e., baseline errors, from the phase of selected pixels in individual interferograms and their subsequent adjustment in a network, yielding quasi-absolute orbit errors for particular acquisitions. The approach is based on the stable parameterisation of baseline errors by $d \dot{B}_{\|}$and $d B_{\perp}$, involving rigorous geometric modelling. The mutual consistency of estimated orbit errors obtained from a sample ENVISAT dataset is better than 0.05 fringes or on the millimetre level in terms of baseline errors, respectively. For quality control on the network level, iterative data snooping has proven its capability to detect and reject outliers.

For the estimation of baseline errors from the interferometric phase, two alternatives are proposed. A least squares estimator supplementarily provides statistical information, which can be useful for an optimal weighting scheme and quality control. However, it requires prior phase unwrapping. This is not the case for the gridsearch approach, which has the drawback of occasionally unreliable estimates.

The most outstanding distinction of the presented method is the mutual controllability of baseline estimates, enhancing resistance to outliers or blunders. A further improvement compared to many existing approaches is the numerically stable parameterisation by $d \dot{B}_{\|}$and $d B_{\perp}$. Topographic variations are fully accounted for, and the bias due to deformation may be mitigated in a combined processing with PS approaches.

Potential improvements of the method would involve a relaxation of the requirement that the observation pixels need to be homogeneously distributed, which might be achievable by robust estimation techniques. Furthermore, all stochastic models analysed so far are still far from optimal in several respects. Nevertheless, it is questionable if the benefit from developing an even more adequate model would outweigh the effort involved. 
Acknowledgments The first author is indebted to the Karlsruhe House of Young Scientists (KHYS) and the Graduate School for Climate and Environment (GRACE) for supporting research stays at Delft University of Technology. We would like to thank Kurt Feigl and two anonymous reviewers for providing detailed and constructive comments that helped improving this contribution.

\section{References}

Baarda W (1968) A testing procedure for use in geodetic networks, vol 9. NCG Publications on Geodesy, Delft

Bähr H, Hanssen RF (2010) Network adjustment of orbit errors in SAR interferometry. In: Lacoste-Francis $\mathrm{H}$ (ed) Proceedings of 'Fringe 2009', Frascati, Italy, 30 Nov-4 Dec 2009. ESA conference proceedings, vol SP-677

Biggs J, Wright T, Lu Z, Parsons B (2007) Multi-interferogram method for measuring interseismic deformation: Denali Fault, Alaska. Geophys J Int 170(3):1165-1179

Bing C, Shaojian X, Ping Z (2006) Estimation of InSAR baseline based on the frequency shift theory. In: Shunjun W (ed) Proceedings of the 2006 CIE international conference on radar, Shanghai, China, 16-19 Oct 2006

Chen CW, Zebker HA (2001) Two-dimensional phase unwrapping with use of statistical models for cost functions in nonlinear optimization. J Opt Soc Am A 18(2):338-351

Doornbos E, Scharroo R (2005) Improved ERS and ENVISAT precise orbit determination. In: Lacoste H, Ouwehand L (eds) Proceedings of the 2004 ENVISAT \& ERS symposium, Salzburg, Austria, 6-10 Sep 2004. ESA conference proceedings, vol SP-572

European Space Agency (2007) Information on ALOS PALSAR products for ADEN users. ESA technical note

Ferretti A, Prati C, Rocca F (2001) Permanent scatterers in SAR interferometry. IEEE Trans Geosci Remote Sens 39(1):8-20

Goldstein RM, Werner CL (1998) Radar interferogram filtering for geophysical applications. Geophys Res Lett 25(21):4035-4038

Hanssen RF (2001) Radar interferometry-data interpretation and error analysis. Kluwer, Dordrecht

Heck B (1981) Der Einfluß einzelner Beobachtungen auf das Ergebnis einer Ausgleichung und die Suche nach Ausreißern in den Beobachtungen. Allg Vermess-Nachr 88(1):17-34

Hooper AJ, Segall P, Zebker HA (2007) Persistent scatterer interferometric synthetic aperture radar for crustal deformation analysis, with application to Volcan Alcedo, Galápagos. J Geophys Res 112:B07407. doi:10.1029/2006JB004763

Jäger R, Müller T, Saler H, Schwäble R (2006) Klassische und robuste Ausgleichungsverfahren. Wichmann, Heidelberg

Kampes BM, Hanssen RF, Perski Z (2004) Radar interferometry with public domain tools. In: Lacoste $\mathrm{H}$ (ed) Proceedings of Fringe 2003 workshop, Frascati, Italy, 1-5 Dec 2003. ESA conference proceedings, vol SP-550

Ketelaar VBH (2009) Satellite radar interferometry—subsidence monitoring techniques. Springer, Berlin

Koch KR (1999) Parameter estimation and hypothesis testing in linear models, 3rd edn. Dümmler, Bonn
Kohlhase AO, Feigl KL, Massonnet D (2003) Applying differential InSAR to orbital dynamics: a new approach for estimating ERS trajectories. J Geod 77(9):493-502

Kreyszig E (1979) Statistische Methoden und ihre Anwendungen, 7th edn. Vandenhoek \& Ruprecht, Göttingen

Lundgren P, Hetland EA, Liu Z, Fielding EJ (2009) Southern San Andreas-San Jacinto fault system slip rates estimated from earthquake cycle models constrained by GPS and interferometric synthetic aperture radar observations. J Geophys Res 114:B02403

Massonnet D, Feigl KL (1998) Radar interferometry and its applications to changes in the earth's surface. Rev Geophys 36(4): $441-500$

Monti Guarnieri A, Biancardi P, D'Aria D, Rocca F (2000) Accurate and robust baseline estimation. In: Proceedings of Fringe '99, Liège, Belgium, 10-12 Nov 1999. ESA conference proceedings, vol SP-478

Nakamura R, Nakamura S, Kudo N, Katagiri S (2007) Precise orbit determination for ALOS. In: 20th International symposium on space flight dynamics, Annapolis, USA, 24-28 Sep 2007

Otten M, Dow JM (2005) ENVISAT precise orbit determination. In: Lacoste H, Ouwehand L (eds) Proceedings of the 2004 ENVISAT \& ERS Symposium, Salzburg, Austria, 6-10 Sep 2004

Pope AJ (1976) The statistics of residuals and the detection of outliers. NOAA technical report no. 65 NGS 1, US Department of Commerce, Rockville, MD, USA

Scharroo R, Visser PNAM (1998) Precise orbit determination and gravity field improvement for the ERS satellites. J Geophys Res 103(C4):8113-8127

Singh K, Stussi N, Keong KL, Hock L (1997) Baseline estimation in interferometric SAR. In: Stein TI (ed) Proceedings of the 1997 international geoscience and remote sensing symposium, IGARSS '97, Singapore, 3-8 Aug 1997

Small D, Werner CL, Nüesch D (1993) Baseline modelling for ERS-1 SAR interferometry. In: Proceedings of the 1993 international geoscience and remote sensing symposium, IGARSS '93: better understanding of Earth environment, Tokyo, Japan, 18-21 Aug 1993, pp 1204-1206

Teunissen PJG (2000) Adjustment theory. Delft University Press, Delft Werner CL, Hensley S, Goldstein RM, Rosen PA, Zebker HA (1993) Techniques and applications of SAR interferometry for ERS-1: topography, change detection and slope measurement. In: Kaldeich B (ed) Proceedings of the first ERS-1 symposium: space at the service of our environment, Cannes, France, 4-6 Nov 1992, pp 205-210

Yoon YT, Eineder M, Yague-Martinez N, Montenbruck O (2009) TerraSAR-X precise trajectory estimation and quality assessment. IEEE Trans Geosci Remote Sens 47(6):1859-1868

Zhang K, Ng AHM, Li X, Chang HC, Ge L, Rizos C (2009) A new approach to improve the accuracy of baseline estimation for spaceborne radar interferometry. In: Proceedings of the IEEE international geoscience and remote sensing symposium, Cape Town, South Africa, 12-17 July 2009 